University of Nebraska - Lincoln DigitalCommons@University of Nebraska - Lincoln

2003

\title{
Grid convergence of high order methods for multiscale complex unsteady viscous compressible flows
}

Björn Sjögreen

NASA Ames Research Center, Bjorn.Sjogreen@multid.se

Helen C. Yee

NASA Ames Research Center, yee@nas.nasa.gov

Follow this and additional works at: http://digitalcommons.unl.edu/nasapub

Sjögreen, Björn and Yee, Helen C., "Grid convergence of high order methods for multiscale complex unsteady viscous compressible flows" (2003). NASA Publications. 239.

http://digitalcommons.unl.edu/nasapub/239

This Article is brought to you for free and open access by the National Aeronautics and Space Administration at DigitalCommons@University of Nebraska - Lincoln. It has been accepted for inclusion in NASA Publications by an authorized administrator of DigitalCommons@University of Nebraska - Lincoln. 


\title{
Grid convergence of high order methods for multiscale complex unsteady viscous compressible flows ${ }^{\text {is }}$
}

\author{
B. Sjögreen ${ }^{a, *}$, H.C. Yee ${ }^{b}$ \\ a Department of Numerical Analysis and Computer Sciences, KTH, 10044 Stockholm, Sweden \\ ${ }^{\mathrm{b}}$ NASA Ames Research Center, Moffett Field, CA 94035, USA
}

Received 23 August 2001; received in revised form 20 September 2002; accepted 27 October 2002

\begin{abstract}
Grid convergence of several high order methods for the computation of rapidly developing complex unsteady viscous compressible flows with a wide range of physical scales is studied. The recently developed adaptive numerical dissipation control high order methods referred to as the ACM and wavelet filter schemes are compared with a fifthorder weighted ENO (WENO) scheme. The two 2-D compressible full Navier-Stokes models considered do not possess known analytical and experimental data. Fine grid solutions from a standard second-order TVD scheme and a MUSCL scheme with limiters are used as reference solutions. The first model is a 2-D viscous analog of a shock tube problem which involves complex shock/shear/boundary-layer interactions. The second model is a supersonic reactive flow concerning fuel breakup. The fuel mixing involves circular hydrogen bubbles in air interacting with a planar moving shock wave. Both models contain fine scale structures and are stiff in the sense that even though the unsteadiness of the flows are rapidly developing, extreme grid refinement and time step restrictions are needed to resolve all the flow scales as well as the chemical reaction scales. Our computations were all made on uniform grids, and our conclusions cannot be directly carried over to, for example, curvilinear grids.

(C) 2002 American Institute of Aeronautics and Astronautics, Inc. Published by Elsevier Science B.V. All rights reserved.
\end{abstract}

\section{Introduction}

There has been much discussion on verification and validation processes for establishing the credibility of CFD simulations $[3,13,27,35,40]$. Since the early 1990 s, many of the aeronautical and mechanical en-

\footnotetext{
No copyright is asserted in the United States under Title 17, US Code. The US Government has a royalty-free license to exercise all rights under the copyright claimed herein for Governmental Purposes. All other rights are reserved by the copyright owner. RIACS Technical Report 01.06, April 2001, NASA Ames Research Center; AIAA 2001-2599, Proceedings of the 15th AIAA CFD Conference, June 11-14, 2001, Anaheim, CA. Part of this work was carried out while the first author was a visiting scientist with the Research Institute of Advanced Computer Science (RIACS), NASA Ames Research Center.

${ }^{*}$ Corresponding author. Fax: +46-8-790-6457.

E-mail address: bjorns@nada.kth.se (B. Sjögreen).
}

0021-9991/02/\$ - see front matter (C) 2002 American Institute of Aeronautics and Astronautics, Inc. Published by Elsevier Science B.V. All rights reserved.

PII: S0021-9991(02)00044-X 
gineering related reference journals mandated that any accepted articles in numerical simulations (without known solutions to compare with) need to perform a minimum of one level of grid refinement and time step reduction. Due to the difficulty in analysis, the effect of under-resolved grids and the nonlinear behavior of available spatial discretizations are scarcely discussed in the literature. Here, an under-resolved numerical simulation is one where the grid spacing being used is too coarse to resolve the smallest physically relevant scales of the chosen continuum governing equations that are of interest to the numerical modeler.

With the advent of new developments in fourth-order or higher spatial schemes, it has become common to regard high order schemes as more accurate, reliable, and requiring less grid points. The danger comes when one tries to perform computations with the coarsest grid possible while still hoping to maintain numerical results sufficiently accurate for complex flows and, especially, data-limited problems. On one hand, high order methods when applied to highly coupled multidimensional complex nonlinear problems might have different stability, convergence and reliability behavior than their well studied low order counterparts, especially for nonlinear schemes such as TVD, MUSCL with limiters, ENO, WENO, and spectral elements and discrete Galerkin, see for example [2,14,26,39,44-47]. On the other hand, high order methods involve more operation counts and systematic grid convergence study can be time consuming and prohibitively expensive. At the same time it is difficult to fully understand or categorize the different nonlinear behavior of finite discretizations, especially at the limits of under-resolution when different types of bifurcation phenomena might occur, depending on the combination of grid spacings, time steps, initial conditions and numerical treatments of boundary conditions (BCs) as well as the nonlinear stability of the scheme in question.

Objective: Grid convergence study of our recently developed adaptive numerical dissipation control high order methods $[33,48,49]$, referred to as the artificial compression method (ACM) and wavelet based filter schemes is the focus of this paper. In a forthcoming paper, by numerical experiments and using tools from dynamical system, some representative nonlinear behavior of high order schemes for under-resolved grids will be discussed (see references [2,12,14,26,39,44-47] for some previous discussions). Two stiff multiscale complex unsteady compressible Navier-Stokes models are chosen for the numerical experiment. The first model is a 2-D viscous analog of a shock tube problem which involves complex shock/shear/boundary-layer interactions. The second model is a supersonic reactive flow concerning fuel breakup. This fuel mixing involves circular hydrogen bubbles in air interacting with a planar moving shock wave. The results are compared with a fifth-order weighted ENO (WENO) scheme. Both models do not have known analytical and experimental data. Fine grid solutions from a standard second-order TVD scheme and a MUSCL scheme with limiters are used as reference solutions. The difficulty in obtaining well resolved multiscale flow structure will be discussed. Note that the present study is confined to uniform grids. At present, efficient and reliable dynamic grid adaptation for rapidly developing multiscale flows are not fully developed. If one were to use standard static adaptive grids (or static adaptive grid refinements), frequent adaptations are expected.

\section{Numerical methods}

Standard stability guidelines for finite difference methods in solving nonlinear fluid flow equations are based on a linearized stability analysis. The linear stability criterion is applied to the frozen nonlinear problem at each time step and grid point. Most often the numerical BC (or boundary scheme) if needed, is not part of the stability analysis. This section gives a brief summary of the recent developments on stable numerical BC treatment for central higher-order interior schemes (schemes for grid points away from the boundaries). These recent developments are for problems containing continuous solutions. The discussion divides into linearly stable and nonlinearly stable difference methods. Since we are solving the NavierStokes equations with complex viscous shock, shear-layer, and boundary layer and/or chemical reaction 
interactions, even after incorporating tools from recent developments, our finite difference method considered in this paper, although more rigorous than standard algorithms in use for practical simulations, is only linearly stable in a strict sense.

\subsection{Linearly stable difference methods}

The most basic linear stability criterion is to investigate the behavior of the difference method when applied to a problem of constant coefficients and periodic boundaries. The Fourier symbol of the operator should be bounded. For higher than first-order methods, a complication is introduced by the numerical boundary treatment. Norm estimates, or normal mode analysis are normally employed (see [15]). With these methods it is possible to prove stability for linear initial boundary value problems (IBVPs). Difference operators having the so-called summation by parts (SBP) property have recently received some attention (see $[4,29-31,36])$. The idea with these operators is to have the property

$$
\left(D u_{j}, v_{j}\right)=-\left(u_{j}, D v_{j}\right)+v_{N} u_{N}-v_{1} u_{1},
$$

where $D$ is a difference operator approximating $\mathrm{d} / \mathrm{d} x$, including the accompanied boundary scheme. Typically $D$ is a standard centered operator in the interior of the computational domain, and has a special one-sided form near boundaries. The scalar product is defined by

$$
(u, v)=\sum_{i, j=1}^{N} \sigma_{i, j} u_{i} v_{j}
$$

with $\sigma_{i, j}$ a positive definite matrix. For the standard 2-norm, $\sigma$ is the identity matrix. In [29,36], formulas for the norm and boundary modifications of $D$ are given which ensure the SBP property for operators up to order of accuracy eight. With the summation by parts property, norm estimates of the difference approximation can be accomplished as the discrete analog of the integration by parts procedure for the continuous energy estimate of the corresponding IBVP of the partial differential equation (PDE). For example, integration by parts of the IBVP of the standard hyperbolic model PDE

$$
u_{t}+a u_{x}=0, \quad 0<x<1
$$

and taking the inner product of the resulting PDE with $u(x, t)$ and integrating will result in the product norm

$$
\frac{1}{2} \frac{\mathrm{d}}{\mathrm{d} t}(u, u)=-\left(u, a u_{x}\right)=a\left(u_{x}, u\right)+a\left[-u^{2}(1, t)+u^{2}(0, t)\right] .
$$

It is trivial to see that

$$
\frac{\mathrm{d}}{\mathrm{d} t}(u, u)=a\left[-u^{2}(1, t)+u^{2}(0, t)\right] \leqslant a u^{2}(0, t),
$$

if we assume $a>0$. The norm is bounded by given data on the inflow boundary. The discrete analog of the same analysis can now be used for the semi-discrete case via the method of lines approach, obtained by discretizing in space, if the difference operator has the SBP property.

A difficulty occurs when the problem is discretized in time. It is possible that the SBP property is destroyed by the time discretization. Several methods stating how to impose the physical BC have been proposed to overcome the difficulty. Examples are the projection method [29] and the penalty method called "simultaneous approximation term" (SAT) [4,5]. For comparison of these methods, see [18,24,37]. In addition, when time-dependent physical BCs are involved, an additional complication arises, especially for 
multi-stage Runge-Kutta methods. If the time-dependent physical BC is not imposed correctly, the overall order of accuracy of the scheme cannot be maintained. Some systematic remedies are proposed but are rather complicated for variable coefficients and even more complicated for nonlinear problems (see $[5,6,18])$. In our computer code, we have implemented the sixth-order SBP operators given in $[4,5,29]$. They both perform satisfactorily, and no big difference in performance has been observed between them. It is noted that the physical BCs of our two models are not time dependent, and the loss of spatial accuracy due to the multistage Runge-Kutta method is not a major concern.

As for how to impose boundary data, the methods given in $[4,5,29]$ are based on linear properties and cannot, except for certain special cases, trivially be generalized to the nonlinear Navier-Stokes equations. One such special case where the nonlinear case is covered by the theory involves imposing velocity zero on solid walls, where the simple approach of setting the velocity to zero after each time step coincides with the projection method in [29].

The SBP operators can be used to show stability for linear problems on symmetric form, as indicated above. The theory does not straightforwardly apply to the fully nonlinear Navier-Stokes equations, which we solve in our computations. However, stability for the linearized problem is a necessary condition for nonlinear stability, since the linearized problem is obtained by considering small perturbations of a constant state for the nonlinear problem. Furthermore, in our computations with the nonlinear Navier-Stokes equations, we do obtain a stable solution when using SBP operators.

\subsection{Nonlinearly stable difference methods}

When using a linearly stable method on a nonlinear problem, nonlinear instabilities can appear. Instabilities can appear already for a linear problems with variable coefficients. For variable coefficient problems, it can be proved that numerical dissipation of not too high order will make the method stable. From a theorem by Strang it follows that a finite difference approximation of a nonlinear problem is stable, if the variable coefficient linearized approximation is stable, and the solution and the difference scheme are smooth functions. This is one reason for using numerical dissipation in practical flow simulation [15].

For certain conservation laws, in the absence of discontinuous solutions, it is possible to achieve nonlinear stability directly without resorting to numerical dissipation. This is done first through a transformation of the PDE, and then through the use of the SBP satisfying interior and boundary difference operators to solve the transformed PDE. For a symmetrizable system of conservation laws, it is possible to transform the PDEs in terms of new variables with the desired property [28]. For the compressible Euler equations, it turns out that the transformed variables can be a function of the physical entropy $[11,17]$, hereafter, referred to as entropy splitting of the PDEs [49]. The entropy splitting splits the inviscid flux derivatives into conservative and non-conservative portions. The idea is a generalization of the following splitting for the quadratic flux function

$$
\left(u^{2} / 2\right)_{x}=\frac{1}{3} u u_{x}+\frac{2}{3}\left(u^{2} / 2\right)_{x} .
$$

For example, introducing the above splitting into the inviscid Burgers' equation

$$
u_{t}+\left(u^{2} / 2\right)_{x}=0
$$

gives, by taking the inner product and integration by parts on the conservative term, the estimate

$$
\frac{\mathrm{d}}{\mathrm{d} t}(u, u)=-\left(\frac{1}{3}\left(u^{2}, u_{x}\right)+\frac{2}{3}\left(u,\left(u^{2} / 2\right)_{x}\right)\right)=u(0, t)^{3} / 3-u(1, t)^{3} / 3 .
$$


If one applies the difference operators that satisfy the SBP principle to the transformed nonlinear PDE, it is possible to prove norm estimates for the discretized operator directly. In [11] it is shown how to generalize the above splitting to the perfect gas compressible nonlinear Euler equations. It was further extended to thermally perfect gases and to generalized coordinates that preserve freestream by Yee et al. [49] and Vinokur and Yee [41].

Entropy splitting is built in as an option in our computer code. For strong shocks, the gain in stability by using the entropy splitting is diminished, see [49] for a study. Since the two chosen model problems contain strong viscous shocks, the entropy splitting is not used for the inviscid fluxes for the computations reported here. Successful examples on the benefit of entropy splitting on flows with long time wave propagations, low speed compressible turbulence and/or weak shocks are shown in [32,49].

\subsection{High order filter difference methods}

In order to filter high frequency producing oscillations due to the non-dissipative and low-dissipative nature of the finite discretizations, the use of the linear filter concept for smooth and/or turbulent flows has been employed for over two decades $[1,10,22,42]$. When discontinuities are present in the solution, linear filter and/or entropy splitting might not be helpful or not applicable. TVD, ENO, and WENO schemes have been shown to work well in a variety of rapidly developing shock-shock interactions that do not involve multiscale physics or long time wave propagations. For multiscale physics that require low dispersive errors, the amount of numerical dissipation built-in in these schemes is not optimal. In addition, analog SBP theory for these schemes are not available. In addition, high order TVD, ENO, and WENO schemes are more computationally expensive than standard high order centered schemes, and have severe limitations on the order of accuracy in the vicinity of the discontinuities and steep gradient regions. The inaccuracy of the numerical solutions can contaminate the entire flow field downstream. Moreover, although, the amount of numerical dissipation is less than linear numerical dissipations, when applied to convection portions of viscous flows, it conflicts with the physical viscosity and can wash out the true physical steep gradient and/ or turbulent structures. Aside from this fact, viscous reacting flows are even more difficult to simulate than non-reacting viscous flows. In the presence of numerical dissipations, even what is believed to be the optimal amount for non-reacting flows might have detrimental effects, e.g., wrong speeds of propagation and/ or spurious traveling waves $[20,21,23]$.

An alternative to these types of shock-capturing schemes for viscous multiscale and long time wave propagation computations is the ACM (artificial compression method) filter scheme described in [48]. Instead of using a linear filter, a high order centered base scheme together with the nonlinear dissipative portion of a shock-capturing scheme is used as the filter. For time-marching to steady-states, a combination of the linear and nonlinear filter is proposed in $[48,49]$.

In the Yee et al. method $[48,49]$ one time step consists of one full time step with a fourth-order or higher accurate non-dissipative spatial base scheme along with a post processing step, where regions of oscillation are detected using a gradient-like ACM detector [16]), and filtered by adding the numerical dissipation portion of a shock capturing scheme (of the inviscid fluxes) at these parts of the solution. Often an entropy split form of the inviscid flux derivatives is used. The entropy splitting of the inviscid flux derivative is considered as a conditioned (or more stable) form of the governing equations as well as for the SBP principle. The idea of the scheme is to have the spatially higher non-dissipative scheme activated at all times and to add the full strength, efficient and accurate numerical dissipation only at the shock layers and steep gradients. Thus, it is necessary to have good detectors which flag the layers, and not the oscillatory turbulent parts of the flow field. While minimizing the use of numerical dissipation away from discontinuities and steep gradients, the ACM filter scheme consists of tuning parameters that are physical problem dependent.

To minimize the tuning of parameters, new sensors with improved detection properties were proposed in Sjögreen and Yee [33]. The new sensors are derived from utilizing appropriate non-orthogonal wavelet 
basis functions, and they can be used to completely switch off the extra numerical dissipation outside shock layers. The non-dissipative spatial base scheme of arbitrarily high order of accuracy can be maintained without compromising its stability at all parts of the domain where the solution is smooth. The wavelet sensor is then obtained by computing the estimated Lipschitz exponent of a chosen physical quantity (or vector) to be sensed on a chosen wavelet basis function. The wavelet sensor can be viewed as dual purpose adaptive methods leading to dynamic numerical dissipation control and improved grid adaptation indicators.

These two high order filter schemes are, hereafter, referred to as the ACM and wavelet filter schemes. This filter approach is particularly important for multiscale viscous flows. The procedure takes the physical viscosity and the reacting terms into consideration since only non-dissipative high order schemes are used as the base scheme. In other words, numerical dissipations based on the convection terms are used to filter the numerical solution at the completion of the full step of the time integration, and only at regions where the physical viscosity is inadequate to stabilize the high frequency oscillations due to the non-dissipative nature of the base scheme.

The method applied to the 2-D conservation law where $U$ is the conservative vector and $F$ and $G$ are the inviscid fluxes,

$$
U_{t}+F(U)_{x}+G(U)_{y}=0
$$

can be described as taking, e.g., one full time step by a Runge-Kutta method on the semi-discrete system

$$
\frac{\mathrm{d} U_{j, k}}{\mathrm{~d} t}=-D_{J} F\left(U_{j, k}\right)-D_{K} G\left(U_{j, k}\right),
$$

where $D_{J}$ and $D_{K}$ are high order finite difference operators, acting in the $j$ - and $k$-direction, respectively. They can be the SBP satisfying higher-order difference operators, for example, in our computations we use the sixth-order accurate

$$
\begin{aligned}
D_{J} F\left(U_{j, k}\right)= & \left(3 F\left(U_{j+3, k}\right)-27 F\left(U_{j+2, k}\right)+135 F\left(U_{j+1, k}\right)-135 F\left(U_{j-1, k}\right)+27 F\left(U_{j-2, k}\right)\right. \\
& \left.-3 F\left(U_{j-3, k}\right)\right) /(180 \Delta x), \\
D_{K} G\left(U_{j, k}\right)= & \left(3 G\left(U_{j, k+3}\right)-27 G\left(U_{j, k+2}\right)+135 G\left(U_{j, k+1}\right)-135 G\left(U_{j, k-1}\right)+27 G\left(U_{j, k-2}\right)\right. \\
& \left.-3 G\left(U_{j, k-3}\right)\right) /(180 \Delta y)
\end{aligned}
$$

in the interior, together with SBP boundary modifications. We here consider a rectangular grid with grid spacing $\Delta x$ and $\Delta y$ and time step $\Delta t$. Denote a full Runge-Kutta step

$$
\widetilde{U}_{j, k}^{n+1}=\operatorname{RK}\left(U_{j, k}^{n}\right) .
$$

After the completion of a full Runge-Kutta step, a filter (post processing) step is applied leading to

$$
U_{j, k}^{n+1}=\widetilde{U}_{j, k}^{n+1}-\lambda_{x}\left(\widetilde{F}_{j+1 / 2, k}-\widetilde{F}_{j-1 / 2, k}\right)-\lambda_{y}\left(\widetilde{G}_{j, k+1 / 2}-\widetilde{G}_{j, k-1 / 2}\right)
$$

with $\lambda_{x}=\Delta t / \Delta x$ and $\lambda_{y}=\Delta t / \Delta y$. The filter numerical fluxes $\widetilde{F}_{j+1 / 2, k}$ and $\widetilde{G}_{j, k+1 / 2}$ act in the $j$ - and $k$-coordinate directions, respectively, and are evaluated on the function $\widetilde{U}^{n+1}$. Note that even when using the one stage forward Euler time stepping, the method is not a standard TVD scheme, since the dissipation is still evaluated on the function $\widetilde{U}^{n+1}$. To make it into a standard TVD method, it would have been necessary to evaluate the dissipation on the function $U^{n}$. 
The $l$ th element of the filter flux in the $x$-direction $\widetilde{f}_{j+1 / 2, k}^{l}$ is a product of a sensor $\omega_{j+1 / 2, k}^{l}$ and a nonlinear dissipation $\phi_{j+1 / 2, k}^{l}$. It is of the form

$$
\widetilde{f}_{j+1 / 2, k}^{l}=\omega_{j+1 / 2, k}^{l} \phi_{j+1 / 2, k}^{l} .
$$

The sensor function $\omega_{j+1 / 2, k}^{l}$ is a product of a physical dependent sensor coefficient and a gradient like ACM detector. The nonlinear numerical dissipation $\phi_{j+1 / 2, k}^{l}$ can be obtained, e.g, from the dissipative portion of a TVD scheme. The numerical flux $h_{j+1 / 2}$ of a TVD scheme can be written (for simplicity written down for a 1-D scalar conservation law)

$$
h_{j+1 / 2}=\frac{1}{2}\left(f\left(u_{j}\right)+f\left(u_{j+1}\right)\right)+\frac{1}{2} \phi_{j+1 / 2},
$$

with the first two terms corresponding to the flux average of a centered difference and $\phi_{j+1 / 2}$ being the numerical dissipation portion of the scheme.

For all the numerical experiments, the numerical dissipation portion of the Harten-Yee scheme is used and have the form for the $x$-direction (with the omission of the $k$ index)

$$
\phi_{j+1 / 2}^{l}=\frac{1}{2} Q\left(a_{j+1 / 2}^{l}\right)\left(g_{j+1}^{l}+g_{j}^{l}\right)-Q\left(a_{j+1 / 2}^{l}+\gamma_{j+1 / 2}^{l}\right) \widetilde{\alpha}_{j+1 / 2}^{l}
$$

with $Q(x)=\sqrt{x^{2}+\epsilon^{2}}$, the entropy satisfying remedy for the scheme with entropy correction parameter $\epsilon$ (not to be confused with the entropy splitting parameter). $a_{j+1 / 2}^{l}$ is the $l$ th characteristic speed evaluated at the Roe's average state in the $x$-direction. $\gamma_{j+1 / 2}^{l}$ is the modified characteristic speed and $g_{j}^{l}$ is a slope limiter which is a function of $\widetilde{\alpha}_{j \pm 1 / 2}^{l}$, the jump in the characteristic variable in the $x$-direction.

A form of the sensor $\omega_{j+1 / 2, k}^{-}$proposed in [48] is

$$
\omega_{j+1 / 2}^{l}=\kappa \max \left(\theta_{j}^{l}, \theta_{j+1}^{l}\right),
$$

where

$$
\theta_{j}=\left|\frac{\left|\widetilde{\alpha}_{j+1 / 2}^{l}\right|-\left|\widetilde{\alpha}_{j-1 / 2}^{l}\right|}{\left|\widetilde{\alpha}_{j+1 / 2}^{l}\right|+\left|\widetilde{\alpha}_{j-1 / 2}^{l}\right|}\right|,
$$

see $[48,49]$ for details. It was shown in [33] that the method can be improved by letting the sensor $\omega_{j+1 / 2}^{l}$ instead be based on a regularity estimate obtained from the wavelet coefficients of the solution. The wavelet analysis gives, for a given function $f$, an estimate of the local Lipschitz exponent $\alpha$, defined as the largest $\alpha$ satisfying

$$
\sup _{h \neq 0} \frac{|f(x+h)-f(x)|}{h^{\alpha}} \leqslant C .
$$

This gives information about the regularity of the function $f$ where small $\alpha$ means poor regularity. For a $C^{1}$ wavelet function $\psi$ with compact support, $\alpha$ can be estimated from the wavelet coefficients, defined as

$$
w_{m, j}=\left\langle f, \psi_{m, j}\right\rangle=\int f(x) \psi_{m, j}(x) \mathrm{d} x,
$$

where

$$
\psi_{m, j}=2^{m} \psi\left(\frac{x-j}{2^{m}}\right)
$$


is the wavelet function $\psi_{m, j}$ on scale $m$ located at the point $j$ in space. This definition gives a so called redundant wavelet, which gives (under a few technical assumptions on $\psi$ ) a non-orthogonal basis for $L^{2}$. It is possible to prove that the coefficients $\max _{j}\left|w_{m, j}\right|$ in a neighborhood of $j_{0}$ decay as $2^{m \alpha}$ as the scale is refined, where $\alpha$ is the Lipschitz exponent at $j_{0}$. In practical computation, we have a smallest scale, determined by the grid size. We evaluate $w_{m, j}$ on this scale, $m_{0}$, and a few coarser scales, $m_{0}+1, m_{0}+2$, and estimate the Lipschitz exponent at the point $j_{0}$ by a least square fit to the line [33]

$$
\max _{j \text { near } j_{0}} \log _{2}\left|w_{m, j}\right|=m \alpha_{j_{0}}+c .
$$

For the numerical experiments, the wavelet coefficient $w_{m, j}$ is computed numerically by a recursive procedure, which is a second-order B-spline wavelet or a modification of Harten's multi-resolution scheme [33]. The computation is done in pressure and density, or field by field in characteristic components. The sensor we use in the computations is

$$
\omega_{j+1 / 2}=\max \left(\tau\left(\alpha_{j}\right), \tau\left(\alpha_{j+1}\right)\right),
$$

where

$$
\tau(\alpha)= \begin{cases}1, & \alpha \leqslant 0.5 \\ 0, & \alpha>0.5\end{cases}
$$

see [33] for details.

The same wavelet sensor can be used as an improved grid adaptation indicator over standard indicators. Finer grids and filters are then used only where the wavelet sensor indicates a low $\alpha$ value. For the numerical experiments, the wavelet decomposition was applied in density and pressure, and the maximum wavelet coefficient of the two components was used. The numerical dissipation is switched on wherever the wavelet analysis gives a Lipschitz exponent less than 0.5 . Increasing this number will reduce oscillations, at the price of reduced accuracy (see [33] for other possibilities).

The nonlinear filter dissipation comes from a second-order accurate TVD method, so that if no switching were used $\left(\omega_{j+1 / 2}^{l}=1\right)$ the order of accuracy would have been reduced to second order. The switch $\theta_{j}$ in the ACM method is of order $\Delta x$ when data is smooth, and will therefore give formal order of accuracy three. In practise, the coefficient $\kappa$ is often taken small, so that the constant in front of the thirdorder error term is very small. With the wavelet sensor, the nonlinear dissipation is completely switched off when the solution is smooth, so that the formal order is equal to the high order of the base scheme. However, the dissipation is switched on near the discontinuities, so that the local order of accuracy varies in the computational domain. Note also that no degeneracy of accuracy near smooth extrema will occur since the dissipation is completely switched off when the solution is smooth.

In Fig. 1 the effect of the wavelet filter is demonstrated. The velocity of the 1-D compressible Euler equations after one time step starting from a jump discontinuity is shown. The curve with dashed line style shows the result after one time step with the sixth-order centered scheme, and the solid curve shows the same solution, after the wavelet filter has been applied. The oscillations are clearly smoothed out.

Central differencing is used for the viscous terms with the order matching the order of the base scheme for the inviscid fluxes. For the reacting flow model, pointwise evaluation of the source terms is adopted. That is, the source terms are evaluated at the grid point $(j, k)$. Although the pointwise evaluation of the source terms is not necessarily the most stable and accurate procedure for inviscid flows [20,21], when physical diffusion is present, no known guidelines are available. 


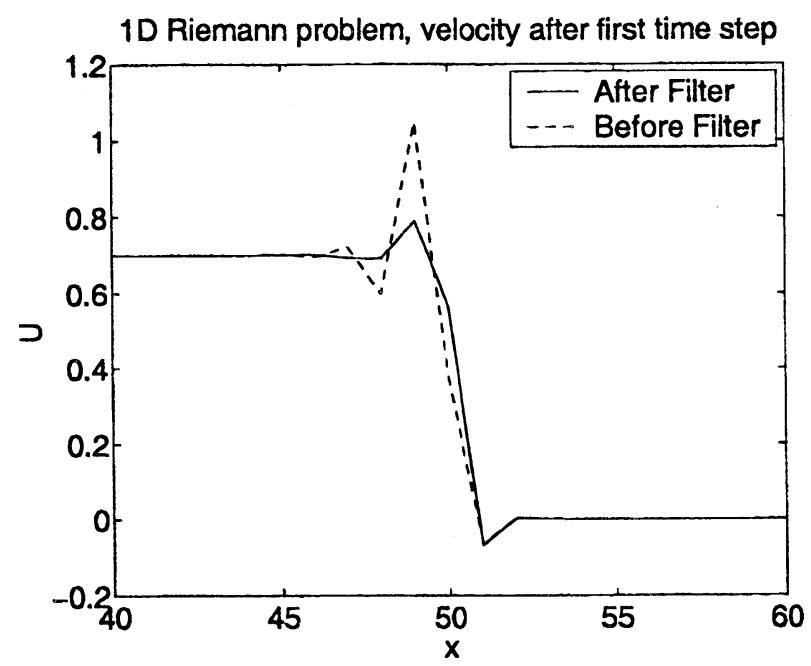

Fig. 1. Spurious oscillation before and after filtering.

\section{A shock/shear/boundary-layer interactions - 2-D viscous shock tube problem}

For the first numerical experiment, the same problem and flow conditions studied in Daru and Tenaud [7] are considered. An ideal gas is at rest in a $2-\mathrm{D}$ box $0 \leqslant x, y \leqslant 1$. A membrane with a shock Mach number of 2.37 located at $x=1 / 2$ separates two different states of the gas. At time zero the membrane is removed and wave interaction occurs. This is a standard shock tube problem, and would give a familiar 1-D wave structure computed by solving a Riemann problem for the inviscid Euler equations. Here, the compressible Navier-Stokes equation with no slip BCs at the adiabatic walls is used. The solution will develop complex 2-D shock/shear/boundary-layer interactions, which depends on the Reynolds number. The complexity of the flow structure increases as the Reynolds number increases.

The dimensionless initial states given in [7] are

$$
\rho_{\mathrm{L}}=120, \quad p_{\mathrm{L}}=120 / \gamma, \quad \rho_{\mathrm{R}}=1.2, \quad p_{\mathrm{R}}=1.2 / \gamma,
$$

where $\rho_{\mathrm{L}}, p_{\mathrm{L}}$ are the density and pressure, respectively, to the left of $x=1 / 2$, and $\rho_{\mathrm{R}}, p_{\mathrm{R}}$ are the same quantities to the right of $x=1 / 2$. All velocities are equal to zero, $\gamma=1.4$ and the Prandtl number is 0.73 . The two Reynolds numbers considered are 200 and 1000. The viscosity is assumed to be constant and independent of temperature, so Sutherland's law is not used. The velocities and the normal derivative of the temperature at the boundaries are set equal to zero. This is done as follows. On each RK stage, the difference scheme is applied in all grid points, including boundary points (by using the one sided SBP boundary modifications), so that the flow field is defined in all points, including boundaries. Next the velocities in the wall boundary points are set equal to zero. For the temperature derivative, a difference operator is used to approximate $\partial T / \partial n=0$, for example

$$
\sum_{k=1}^{p} \alpha_{k} T_{k}=0
$$

where we assume that the boundary is at $k=1$, and $\alpha_{k}$ are the coefficients of a difference operator approximating the derivative at $k=1$, and which has width $p$. We have implemented a sixth-order operator with a seven point wide stencil, but in some cases we were forced, due to stability, to replace it by a second-order, 
three point wide operator. From this we obtain the boundary temperature $T_{1}$ from the interior temperatures $T_{2}, \ldots, T_{p}$. $T_{1}$ is proportional to $p_{1} / \rho_{1}$, and we adjust the boundary pressure to make the boundary temperature computed from $p_{1} / \rho_{1}$ equal to the given $T_{1}$. The boundary density is left unchanged.

With these data an expansion wave and a shock are formed initially. After reflection at the right wall, a left going shock wave appears. The flow is highly unsteady. In the initial phase a boundary layer is formed on the lower boundary behind the right going waves. After reflection, the left going shock wave interacts with the newly formed boundary layer, causing a number of vortices and a lambda shock at the boundary layer. Other kinds of layers remain after the shock reflection near the right wall.

All the computations stop at the dimensionless time 1 when the reflected shock wave has almost reached the middle of the domain, $x=1 / 2$. All the numerical results discussed here are at time 1 with uniform Cartesian grid spacings as was done by Daru and Tenaud. Due to symmetry only the lower half of the domain is used in the computations. Symmetry BCs are enforced at the boundary $y=1 / 2$.

\subsection{Viscous shock tube numerical results, $R e=200$}

We first show results from computations with Reynolds number 200. In order to have a very accurate reference solution, we solved the problem on grids of increasing refinement using a second-order MUSCL scheme with the van Albada limiter applied to the characteristic variables for inviscid flux derivatives, and second-order central for the viscous flux derivatives. The temporal discretization is a second-order accurate Runge-Kutta method. We denote this scheme as MUSCL-RK2. The results are shown in Fig. 2 where the number of grid points are $250 \times 125,500 \times 250,1000 \times 500,2000 \times 1000$, and $3000 \times 1500$, respectively. Density contours at time 1 are shown. Contour values are the same for all figures. To enhance viewing, the plotting region concentrates only on $0.4 \leqslant x \leqslant 1,0 \leqslant y \leqslant 0.3$.

The solutions for $1000 \times 500$ points and $2000 \times 1000$ points are seen to be almost identical, and we conjecture that grid convergence has been reached for the finest resolution. Furthermore, inspecting 1-D cuts in the $2000 \times 1000$ solution reveals that all layers are resolved with at least 5-10 grid points, including the point where a very sharp layer hits the lower wall at $x=0.8$. The only exception is the main shock, which is resolved with only two grid points, even at the finest grid.

Another indication of the quality of the solution is given in Fig. 3, where the solution is computed on $1000 \times 500$ grid points, but with the standard second-order centered difference operator for both the inviscid and viscous flux derivatives, and a second-order Runge-Kutta temporal discretization (CEN22$\mathrm{RK} 2$ ). In this computation, no numerical dissipation is added with only the physical dissipation coming from the Navier-Stokes equations. The only feature where spurious oscillations appear is at the main shock wave. All other structures seem to be well resolved. In view of this cross check, we accept the result from the MUSCL computation on $2000 \times 1000$ (also $3000 \times 1500$ ) points as a reliable reference solution. It also agrees well with the fine grid $(1000 \times 500)$ result of Daru and Tenaud using a third-order shock-capturing method.

Next, we solve the problem using high order difference methods with the goal of answering the following questions. Will high order give us faster grid convergence than what was seen with the second-order TVD scheme in Fig. 2? If we can use a coarser grid, will we gain in CPU time when we consider the higher computational cost of the higher-order methods? Fig. 4 shows a comparison of the reference computation, ACM66 with an ACM coefficient of $\kappa=0.7$ (sixth-order central base scheme for both the inviscid and viscous flux derivatives), and WENO5 (fifth-order WENO for the inviscid flux derivatives and sixth-order central for viscous flux derivatives). The ACM66 method is post processed by the nonlinear TVD filter after each time step. The method is described in Section 2. The same computations were performed using the WAV66 (i.e., an ACM66 but with the ACM sensor replaced by the wavelet sensor). Similar grid convergence and accuracy as the ACM66 were obtained (figures not shown). For the WAV66, the simplest possible wavelet and a cut off Lipschitz exponent of 0.5 were used. For the ACM66, WAV66 and WENO5, 


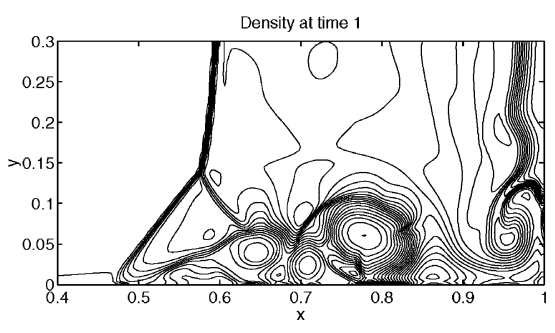

(a)

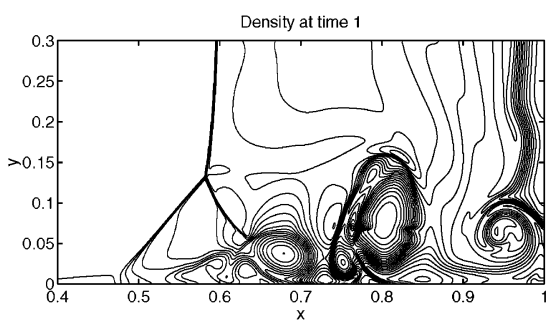

(c)

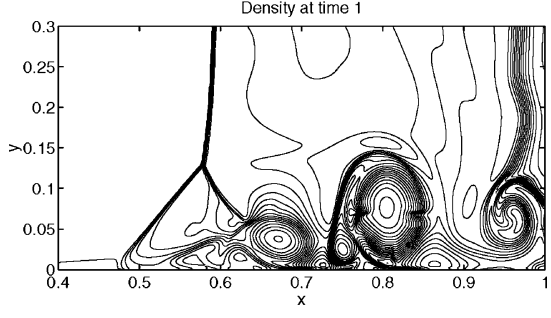

(b)

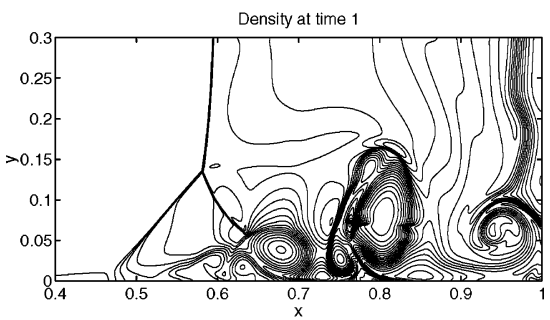

(d)

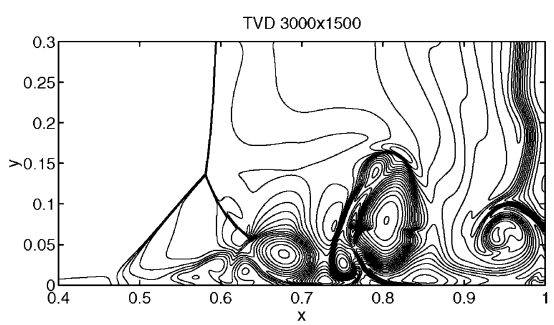

(e)

Fig. 2. Grid refinement and reference solution for $R e=200$ using the MUSCL-RK2 scheme. Density contours of a 2-D shock/shear/ boundary-layer interaction. (a) MUSCL-RK2, $250 \times 125$ grid; (b) MUSCL-RK2, $500 \times 250$ grid; (c) MUSCL-RK2, $1000 \times 500$ grid; (d) MUSCL-RK2, $2000 \times 1000$ grid; (e) MUSCL-RK2, $3000 \times 1500$ grid.

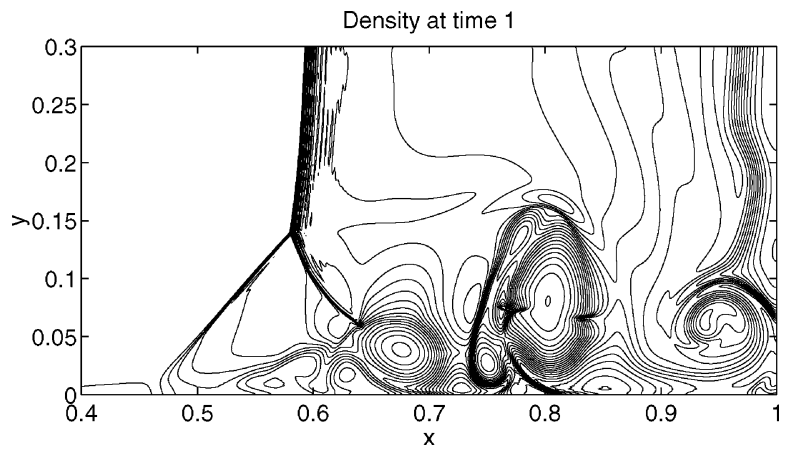

Fig. 3. Density contours using a second-order centered difference (CEN22-RK2) scheme for $R e=200$ on a $1000 \times 500$ grid. 


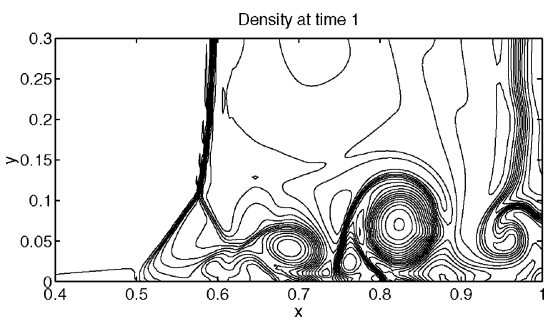

(a)

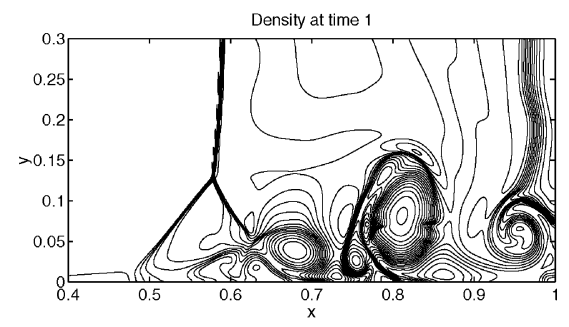

(c)

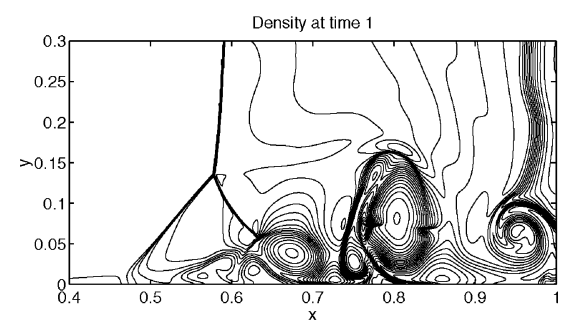

(e)

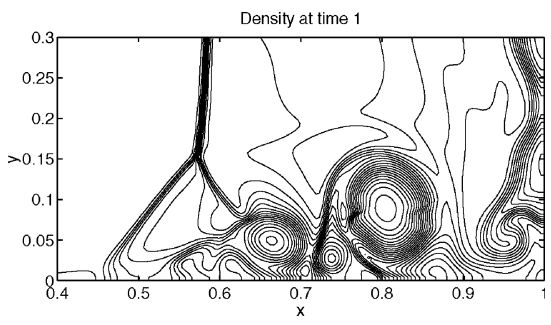

(b)

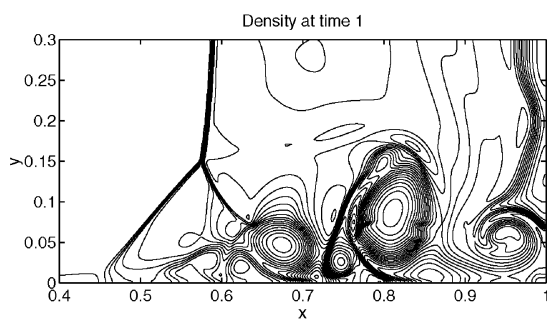

(d)

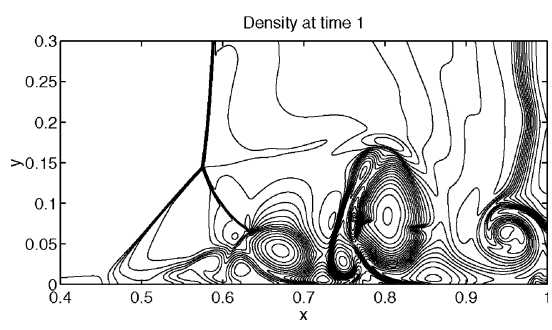

(f)

Fig. 4. Grid refinement comparison of ACM66-RK4 and WENO5-RK4 for Re $=200$. Density contours. (a) ACM66-RK4, $250 \times 125$ grid; (b) WENO5-RK4, $250 \times 125$ grid; (c) ACM66-RK4, $500 \times 250$ grid; (d) WENO5-RK4, $500 \times 250$ grid; (e) ACM66-RK4, $1000 \times 500$ grid; (f) WENO5-RK4, $1000 \times 500$ grid.

the standard fourth-order Runge-Kutta temporal discretization is employed. We denote these schemes as ACM66-RK4, WAV66-RK4 and WENO5-RK4.

The WENO5 is implemented as described in [19] with the only difference being that we use the classical fourth-order Runge-Kutta method in time (WENO5-RK4). The WENO5 method is a discretization of the convective terms using a weighted average of third-order accurate finite difference stencils. The stencils are applied in characteristic variables, and are biased in the upwind direction. The weights in the averaging are made such that stencils over non smooth regions are weighted out, and such that the method becomes fifthorder accurate when the solution is smooth. We used global Lax-Friedrichs fluxes as the basis for the WENO method.

By inspecting Fig. 4, the correct solution is seen to be reached at $500 \times 250$ grid points for the more accurate ACM66-RK4 and WENO5-RK4 methods, one level coarser than the MUSCL scheme. When examining these results more closely, even though WENO5-RK4 predicted the same overall flow structure as ACM66-RK4 using the same grid, the location of the Mach stem and the sizes of the two vortical 
structures are bigger than for ACM66-RK4, the reference solution (MUSCL-RK4 and CEN22-RK4) and the result of Daru and Tenaud, using the $1000 \times 500$ grid. All of the computations shown use the same flux limiter (aside from CEN22-RK2). Due to the high cost of computing, to safeguard against numerous tests for optimal CFL for each method and each grid size, a conservative CFL of 0.4 is used for all the methods and grids. Using a CFL $=0.4$, the MUSCL-RK2 requires 231,745 time steps to reach time 1 on a $3000 \times 1500$ grid. The ACM66-RK4 requires 103,481 time steps, and WENO5-RK4 requires 103,739 time steps to reach to the same time on a $1000 \times 500$ grid. The CPU per time step by WENO-RK4 is 2.5 times the rest of the schemes which are similar in CPU per time step. Therefore, computations beyond a $1000 \times 500$ grid using WENO5-RK4 are too time consuming and costly. It can be argued that the MUSCLRK2 scheme can be made more efficient than the ACM66-RK4 scheme because a larger CFL number can be used for the lower order scheme, so that fewer time steps are needed. However, the practical CFL limit for MUSCL-RK2 is below 1 due to the influence of flux limiters, so the gain is not more than a factor of two, which is, although an improvement, not sufficient to completely compensate for the need for increased grid resolution with the lower order scheme. Computer implementation and computing time comparisons are discussed in Section 5.

\subsection{Viscous shock tube numerical results, $R e=1000$}

We next increase the Reynolds number to 1000 and perform the same computation. For this Reynolds number we are not certain that the flow is stable, see Daru and Tenaud and references cited therein. The flow is very complicated, and one could argue that there is too much uncertainty in the computed results to be of interest. However, on the finest grids we use, the solution appears to have grid converged. Furthermore, the problem has been solved previously in [7], so it is of interest to have a comparison. We hope that our converged solutions can be used for future reference. For example, it is a challenge for adaptive methods to reproduce these solutions at a lower cost.

Again, all of the computations use a fixed CFL of 0.4. The flow is now more complex, and the MUSCLRK2 scheme will require even more grid points to capture the small scales correctly. A sequence of grid refinements is shown in Fig. 5. The finest resolution we used was $4000 \times 2000$ grid points. Due to constraints on computational time, we could not increase the resolution further. Using a CFL $=0.4$, the MUSCL-RK2 requires 106,640 time steps to reach time 1 on a $4000 \times 2000$ grid. The WAV66-RK4 requires 31738 time steps, and WENO5-RK4 requires 32498 time steps to reach to the same time on a $1000 \times 500$ grid. A comparison between high order methods are shown in Figs. 6-8. Comparing the $1000 \times 500$ results, all of the Mach stems are not at the same location. However, the ACM66-RK4 and WENO5-RK4 seem to predict vortical structures that are closer to the result of the MUSCL-RK2 reference solution $(4000 \times 2000$ grid). More remarkable is that the WAV66-RK4 using a $1000 \times 500$ grid predicts the flow structure nearly the same as three to four times (each spatial direction) the grid resolution required by the MUSCL-RK2. Increasing the resolution in the WAV66-RK4 method, gives a few vortices in the layer at $x=0.9$. In [7] such vortices disappear with increased resolution. It is unclear whether they are a spurious numerical effect, or real physical. Although the global structure of the present result is the same as Daru and Tenaud, the fine scale structure is not the same. It appears that the fine scale structure of the Daru and Tenaud results, although using the same grids $(1000 \times 500,2000 \times 1000)$, has not yet converged. The MUSCL-RK2 indicates the general trend of grid convergence. It is surprising to observe that the WENO5RK4 produces results which depart from the rest of the scheme studied (location and shape of the Mach stem and the size of the vortical structure) for both Reynolds numbers. The ACM66-RK4 and WAV66RK4 are not stable for the $500 \times 250$ grid. It is difficult to judge or conclude the performance of the various schemes for $R e=1000$ since we are not certain that this flow is stable. The nearly convergent solutions by the MUSCL-RK2 might be as well due to the more dissipative nature of the scheme and might not be physical in a straight sense. 


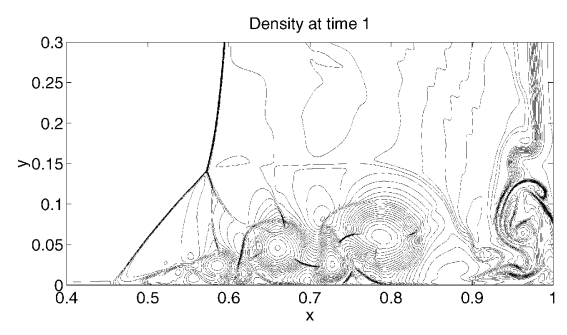

(a)

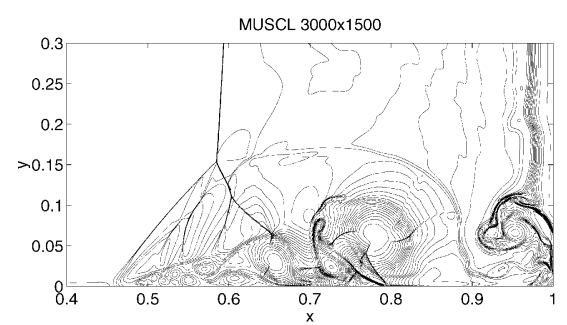

(c)

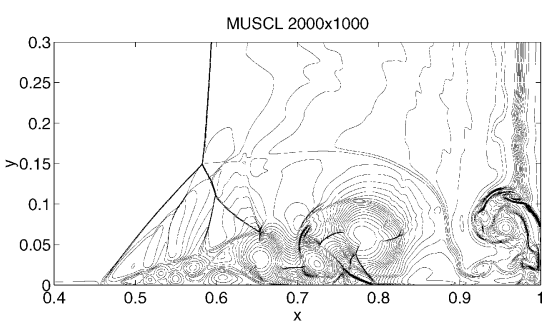

(b)

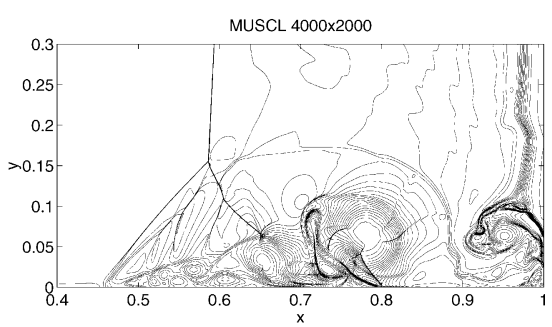

(d)

Fig. 5. Grid refinement and reference solution for $R e=1000$ using the MUSCL-RK2 scheme. Density contours of a 2-D shock/shear/ boundary-layer interaction. (a) MUSCL-RK2, $1000 \times 500$ grid; (b) MUSCL-RK2, $2000 \times 1000$ grid; (c) MUSCL-RK2, $3000 \times 1500$ grid; (d) MUSCL-RK2, $4000 \times 2000$ grid.

To further investigate the grid convergence properties, we display in Fig. 9 the density along the lower wall on grids of increasing refinement. Two methods are compared, MUSCL-RK2 and WAV66RK4. The grids used are $1000 \times 500,2000 \times 1000$, and $3000 \times 1500$. It can be seen that the formally

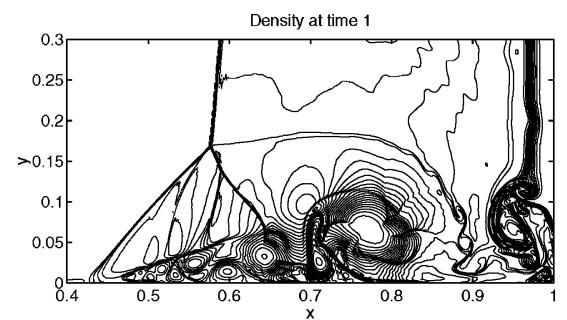

(a)

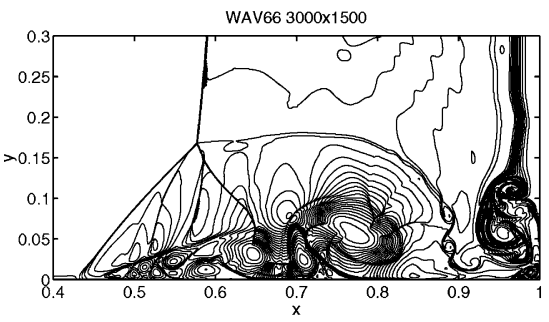

(c)

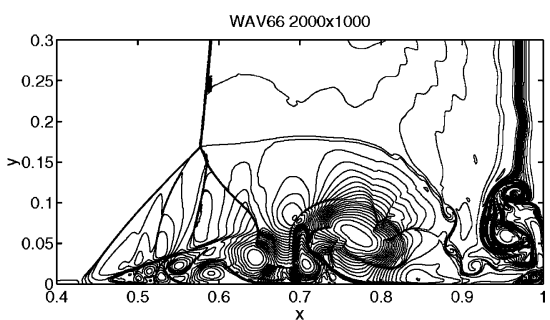

(b)

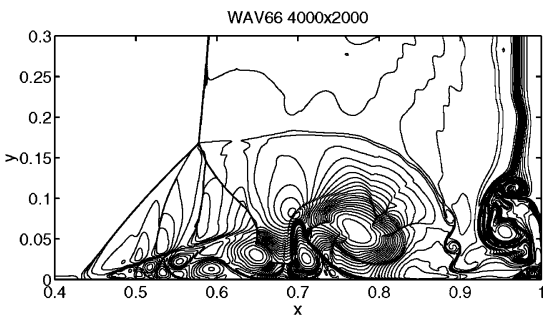

(d)

Fig. 6. Grid refinement of WAV66-RK4 for $R e=1000$. Density contours. (a) WAV66-RK4, $1000 \times 500$ grid; (b) WAV66-RK4, $2000 \times 1000$ grid; (c) WAV66-RK4, $3000 \times 1500$ grid; (d) WAV66-RK4, $4000 \times 2000$ grid. 


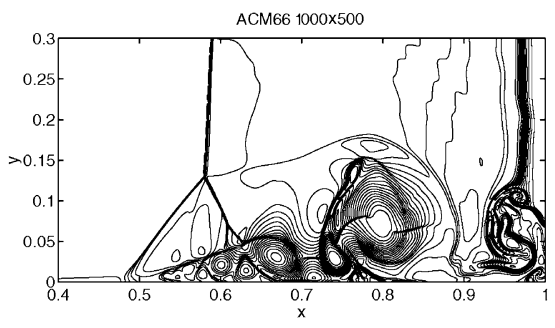

(a)

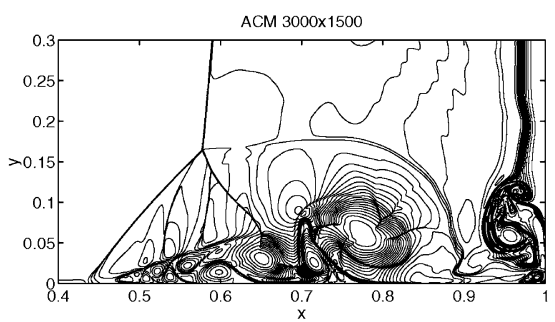

(c)

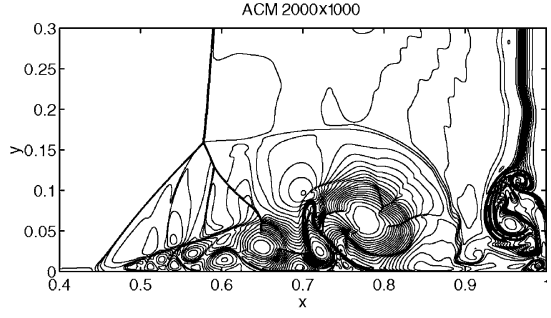

(b)

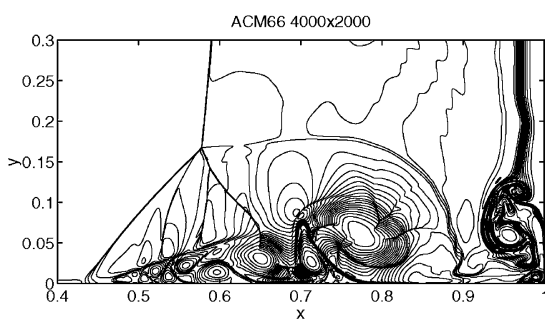

(d)

Fig. 7. Grid refinement of ACM66-RK4 for Re=1000. Density contours. (a) ACM66-RK4, $1000 \times 500$ grid; (b) ACM66-RK4, $2000 \times 1500$ grid; (c) ACM66-RK4, $3000 \times 1500$ grid; (d) ACM66-RK4, $4000 \times 2000$ grid.

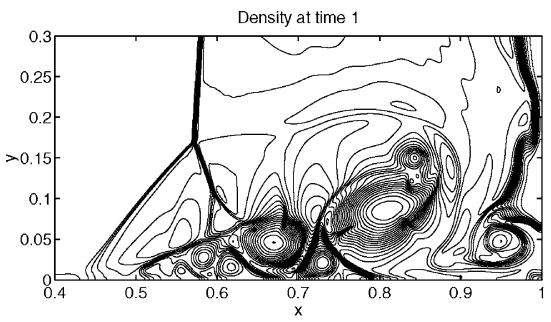

(a)

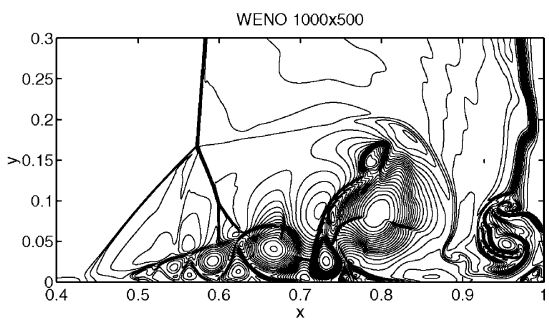

(b)

Fig. 8. Grid refinement of WENO5-RK4 for $R e=1000$. Density contours. (a) WENO5-RK4, $500 \times 250$ grid; (b) WENO5-RK4, $1000 \times 500$ grid .

lower order MUSCL scheme gives a larger difference between the curves than the more accurate WAV66-RK4.

A pointwise convergence study is not possible, since the number of grid points is even, so that the meshes have no common points (excepting the four corner points). Furthermore it is questionable for this complicated flow, to assume that the pointwise error is on the form $C \Delta x^{p}$.

\section{A supersonic combustion model}

In the modeling of viscous problems containing finite-rate chemistry, often a wide range of space and time scales is present due to the reacting terms, over and above the different scales associated with viscous flows, leading to additional numerical difficulties. This stems mainly from the fact that the majority of 


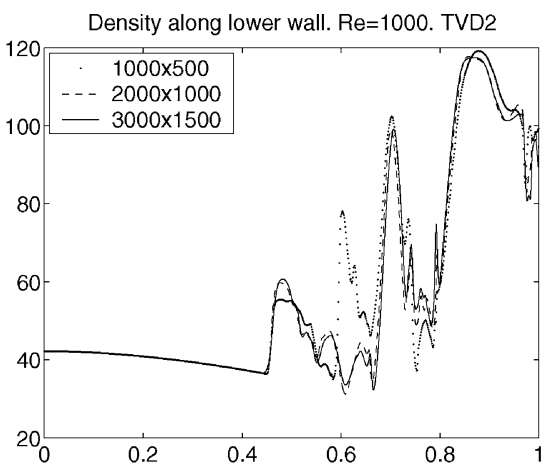

(a)

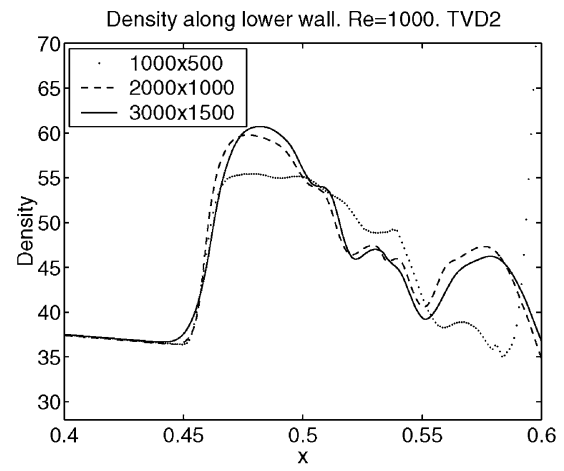

(c)

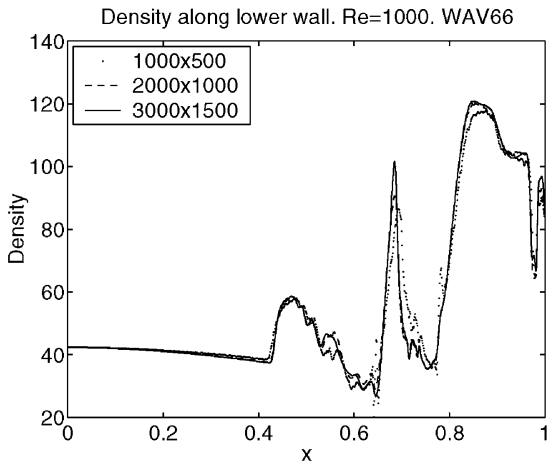

(b)

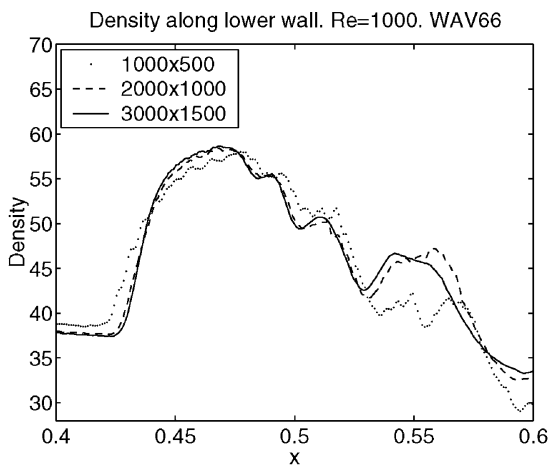

(d)

Fig. 9. Density along lower wall on different grids for $R e=1000$. (a) MUSCL-RK2; (b) WAV66-RK4; (c) MUSCL-RK2, zoomed in part of (a); (d) WAV66-RK4, zoomed in part of (b).

widely used numerical algorithms in reacting flows were originally designed to solve non-reacting fluid flows. When physical diffusion is added, it is not known what type of numerical difficulties will surface.

For the numerical experiment, the same supersonic reactive flow problem concerning fuel breakup and mixing of different configurations as presented in Don and Quillen [8] and Don and Gottlieb [9] is used. This is a simplified model that might be used as fuel mixing in advanced aerospace propulsion systems, e.g., a hydrogen fuel supersonic combustion scramjet that is capable of propelling a space vehicle at hypersonic speed, see [9] and references cited therein for a discussion.

The governing equations are the compressible Navier-Stokes equations with four species undergoing multichemical reactions. The chemical reaction is modeled by a single-step reversible reaction using $\mathrm{H}_{2}, \mathrm{O}_{2}$, $\mathrm{H}_{2} \mathrm{O}$, and $\mathrm{N}_{2}$. A Prandtl number, $P r=0.72$, Schmidt number $S c=0.22$, and the perfect gas equation of state approximation are used. The mixture specific heat at constant pressure was obtained from McBride et al. [25]. The Svehla [38] species viscosity constants and the Wilke's law model [43] for the mixture viscosity are used.

A 2-D flow consisting of a planar shock in air interacting with a circular zone of hydrogen bubbles in two different initial configurations is considered. The two initial configurations are (a) a single bubble and (b) two non-aligned bubbles. The temperature of the hydrogen and air in the undisturbed region ahead of the shock is set to $1000 \mathrm{~K}$ with a pressure of $1 \mathrm{~atm}$. and zero velocity. A Mach 2 shock is placed at $x_{s}=0.005$. The gradient in pressure across the shock in conjunction with the gradient in fluid density 
between the air and hydrogen produce a large increase in vorticity as the shock passes through the hydrogen fuel. As can be seen in the study of Don and Quillen and Don and Gottlieb and the present grid convergence study, the size, spacing and velocity of the vortical structures are very difficult to accurately simulate numerically.

The equations are formulated in the conserved variables $\mathbf{U}=\left(\rho_{1}, \ldots, \rho_{N}, \rho u, \rho v, e\right)$, where $\rho$ is the density of the mixture, $(u, v)$ are velocities, and $e$ is the total energy. $\rho_{i}=\rho y_{i}$, where $y_{i}$ is the mass fraction of species $i$. The equations are in the form

$$
\mathbf{U}_{t}+\mathbf{F}_{x}(\mathbf{U})+\mathbf{G}_{y}(\mathbf{U})=\mathbf{F}_{x}^{v}\left(\mathbf{U}, \mathbf{U}_{x}, \mathbf{U}_{y}\right)+\mathbf{G}_{y}^{v}\left(\mathbf{U}, \mathbf{U}_{x}, \mathbf{U}_{y}\right)+\mathbf{S}(\mathbf{W})
$$

with standard convective flux vectors $\mathbf{F}$ and $\mathbf{G}$, and diffusive flux vectors $\mathbf{F}^{v}$ and $\mathbf{G}^{v}$. $\mathbf{S}(\mathbf{W})$ is the source term representing chemical reactions.

Total energy is modeled as

$$
e=\sum_{i=1}^{N} \rho_{i} \epsilon_{i}(T)+\frac{1}{2} \rho u^{2}+\sum_{i=1}^{N} h_{i}^{0} \rho_{i},
$$

where the internal energy $\epsilon_{i}(T)$ is computed by a polynomial fit to thermodynamical data. The formation enthalpies, $h_{i}^{0}$, are given numbers. Sutherland's law,

$$
\mu_{i}=\mu_{0}^{i}\left(\frac{T}{T_{0}^{i}}\right)^{3 / 2}\left(\frac{T_{0}^{i}+S^{i}}{T+S^{i}}\right),
$$

is used to model the viscosity of each species. $\mu_{0}^{i}, T_{0}^{i}, S^{i}$ are constants, different for different species. The total viscosity of the mixture is computed from Wilke's law,

$$
\mu=\sum_{i=1}^{N} \frac{\mu_{i} X_{i}}{\sum_{j=1}^{N} X_{j} \phi_{i j}},
$$

where

$$
\phi_{i j}=\frac{\left(1+\left(\left(\mu_{i} / \mu_{j}\right)\left(y_{j} / y_{i}\right)\right)^{1 / 2}\left(M_{i} / M_{j}\right)^{1 / 4}\right)^{2}}{\left(8\left(1+M_{i} / M_{j}\right)\right)^{1 / 2}}
$$

and $X_{i}$ are molar fractions, and $M_{i}$ is the molar mass of species $i$. The single-step reversible reaction using $\mathrm{H}_{2}, \mathrm{O}_{2}, \mathrm{H}_{2} \mathrm{O}$, and $\mathrm{N}_{2}$ is

$$
2 \mathrm{H}_{2}+\mathrm{O}_{2} \rightleftharpoons 2 \mathrm{H}_{2} \mathrm{O} \text {. }
$$

Species $\mathrm{N}_{2}$ is inert.

\subsection{Planar shock interacting with a single hydrogen bubble}

In the first test problem a planar shock in air is interacting with a single circular hydrogen bubble. The radius of the hydrogen bubble is 0.01 . A Mach 2 shock is placed at $x_{s}=0.005$. The domain is $0 \leqslant x \leqslant 0.0175$ and $-0.045 \leqslant y \leqslant 0.045$. All of the computations use uniform Cartesian grid spacing as was done by Don and Gottlieb. Fig. 10 shows snap shots of the density at six different stages of the evolutionary process, computed by ACM66-RK4 using a $500 \times 250$ grid. The shock breaks up the bubble into two smaller hydrogen bubbles. These bubbles start to rotate after the shock has passed through, and complex structure is developed inside the bubbles. In Fig. 11, we show a convergence 

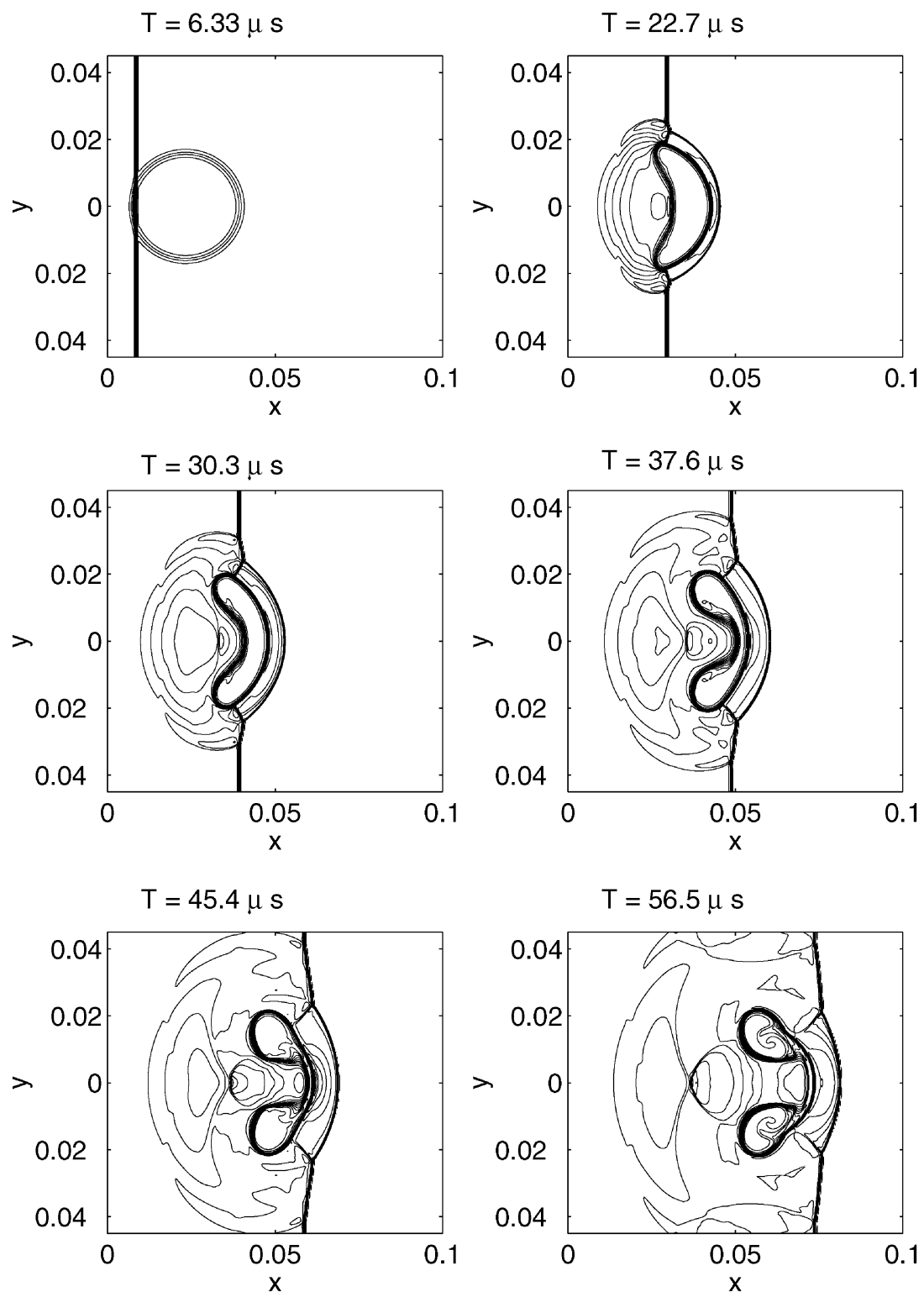

Fig. 10. Density contours of ACM66-RK4 on a $500 \times 250$ grid: Time evolution of a Mach 2 shock in air interacting with one hydrogen bubble.

study at time $60 \mu \mathrm{s}(\mu \mathrm{s})$, using the second-order accurate TVD scheme by Harten and Yee (TVD-RK2). For this study we only compute on the domain $0 \leqslant x \leqslant 0.09$ and $0 \leqslant y \leqslant 0.045$. Symmetry conditions are enforced on the lower boundary. This means setting the velocity normal to the wall equal to zero, and for all other variables setting the normal derivative equal to zero. The numerical approximation is made in the same way as $\partial T / \partial n=0$ is approximated in the shock/shear/boundary layer problem, described previously.

Hydrogen mass fraction is plotted. 


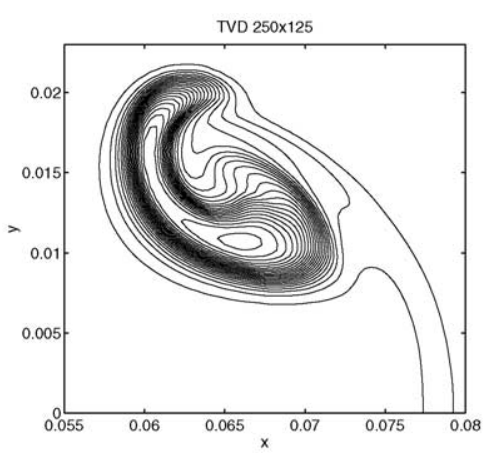

(a)

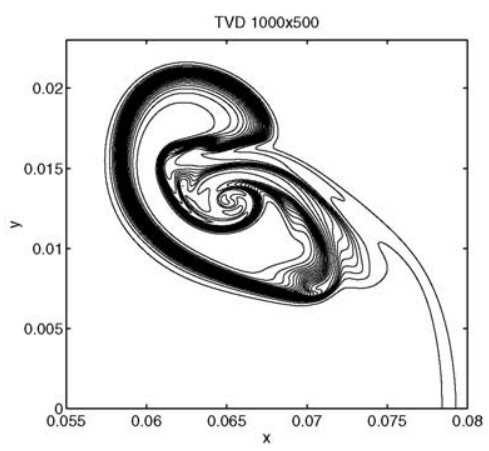

(c)

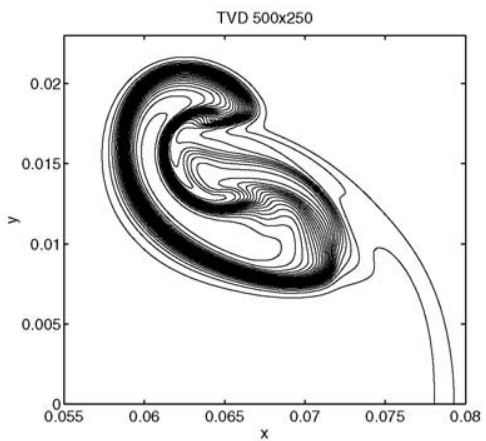

(b)

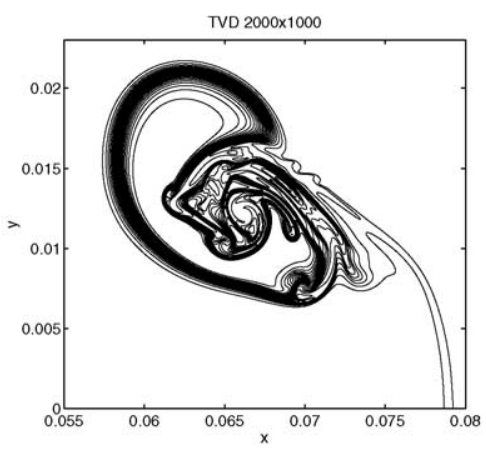

(d)

Fig. 11. Grid refinement of the second-order Harten-Yee TVD scheme (TVD-RK2). Hydrogen mass fraction contours at time $60 \mu$ s. (a) TVD-RK2, $250 \times 125$ grid; (b) TVD-RK2, $500 \times 250$ grid; (c) TVD-RK2, $1000 \times 500$ grid; (d) TVD-RK2, $2000 \times 1000$ grid.

Results from TVD66-RK4, ACM66-RK4 and WENO5-RK4 methods are shown in Figs. 12-14. Here TVD66-RK4 differs from ACM66-RK4 in that the ACM sensor is set to 1, i.e., all the $\kappa \omega_{j+1 / 2}^{l}$ are set to 1 . One can see the advantage of the ACM sensor by examining the two solutions. The results using ACM66-RK4 are less diffusive than those using TVD66-RK4. It seems that WENO5RK4 gives a solution which is similar to the solution obtained by the TVD-RK2 on a grid which is one level finer. The flow structures from ACM66-RK4 and TVD-RK2 are very different. However, the flow structures from the ACM66-RK4 and TVD66-RK4 are similar and have similar convergence trends. Although grid convergence is not perfectly reached with either method, we reach at approximately $2000 \times 1000$ grid points the regime where the viscous, parabolic part of the operator starts to have significant influence on the explicit time step from the CFL condition. For coarser grids we see that the WENO5-RK4 scheme gives a resolution which is similar to that of the ACM66-RK4 scheme, but is somewhat more diffuse. The fine scale solution structures depart from the two methods as we refine the grid. There are two CFL conditions used here, one for the fluid $(\mathrm{CFL}=0.6)$ and one for the reacting terms $(\mathrm{CFL}=0.3)$. The time step is based on the two CFL's, whichever is the smallest. For this model and the above grid convergence study, it is difficult to assess the accuracy and robustness of these schemes. The results reported here reveal the challenge in obtaining the well resolved multiscale complex flow structure of reactive and/or combustion problems. 


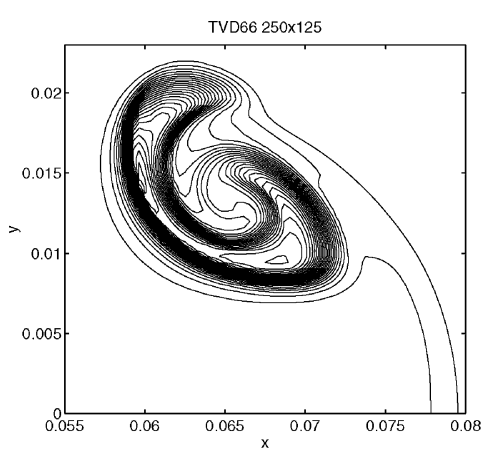

(a)

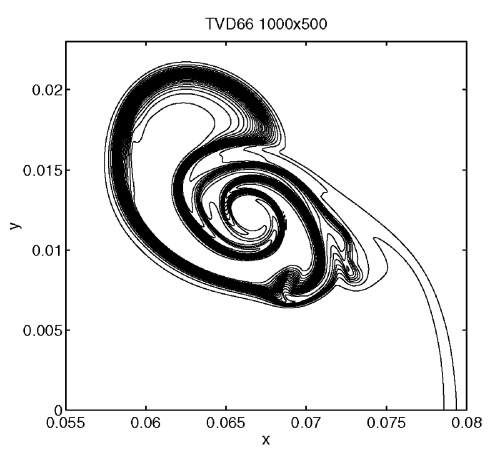

(c)

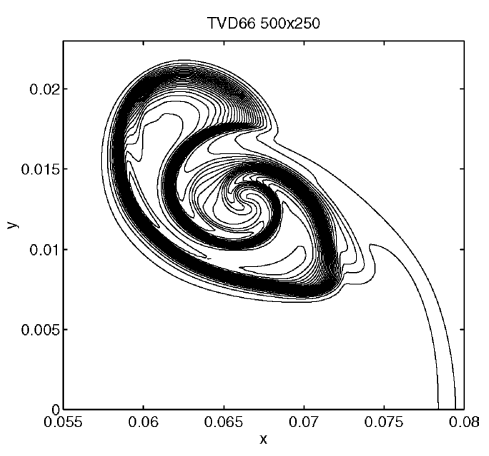

(b)

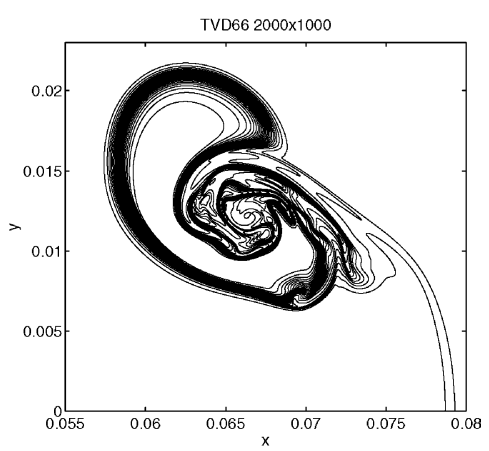

(d)

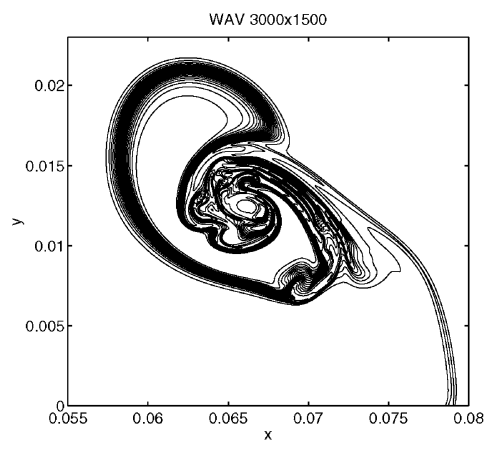

(e)

Fig. 12. Grid refinement of the TVD66-RK4 scheme. Hydrogen mass fraction contours at time $60 \mu$ s. (a) TVD66-RK4, $250 \times 125$ grid; (b) TVD66-RK4, $500 \times 250$ grid; (c) TVD66-RK4, $1000 \times 500$ grid; (d) TVD66-RK4, $2000 \times 1000$ grid; (e) TVD66-RK4, 3000 × 1500 grid.

\subsection{Planar shock interacting with two hydrogen bubbles}

As a second test problem we solve the above equations with initial data consisting of two non-aligned circular hydrogen bubbles. The first is centered at $(0.0275,0.01)$ and the second at $(0.0675,-0.01)$. They both have radius 0.02 . We compute on a domain $0 \leqslant x \leqslant 0.18$, and $-0.045 \leqslant y \leqslant 0.045$. The time evolution 


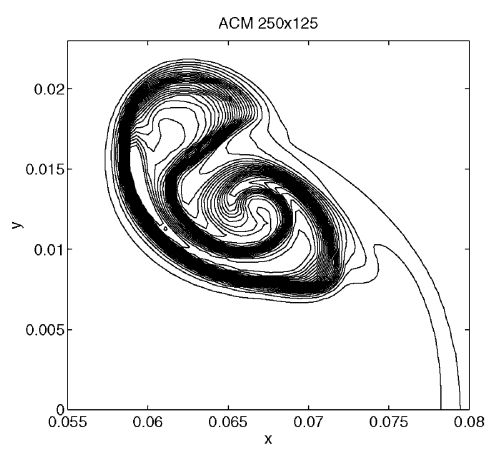

(a)

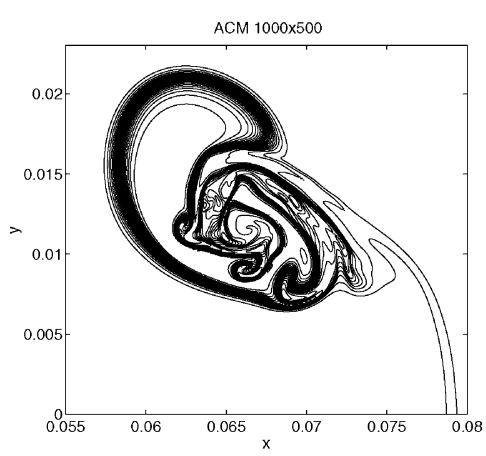

(c)

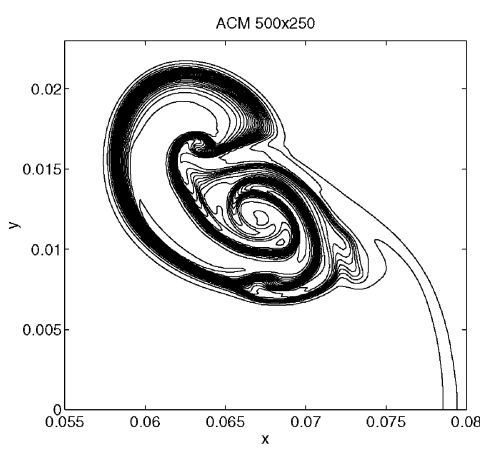

(b)

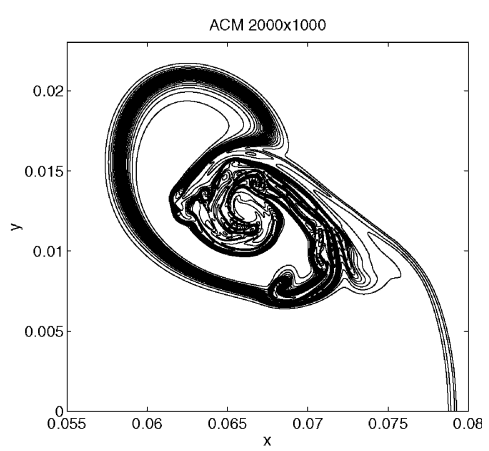

(d)

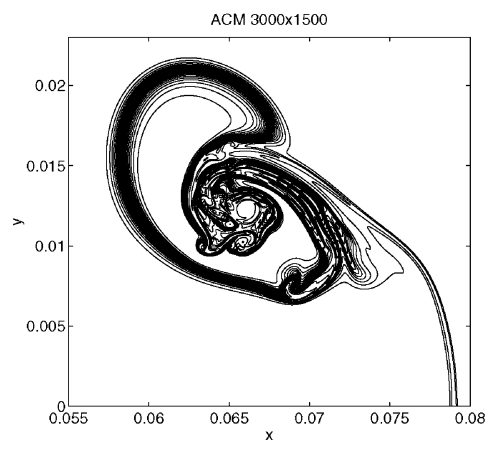

(e)

Fig. 13. Grid refinement of the ACM66-RK4 scheme. Hydrogen mass fraction contours at time $60 \mu$ s. (a) ACM66-RK4, $250 \times 125$ grid; (b) ACM66-RK4, $500 \times 250$ grid; (c) ACM66-RK4, $1000 \times 500$ grid; (d) ACM66-RK4, $2000 \times 1000$ grid; (e) ACM66-RK4, $3000 \times 1500$ grid.

of the flow up to time $125 \mu$ s is shown in Fig. 15. The asymmetric nature of the initial data causes the hydrogen bubbles to split up into smaller pieces. The flow pattern is very complicated. For details see [9]. A similar grid convergence study was performed. For the same grid sizes, grid convergence was reached and fine scale structure inside the hydrogen bubbles was resolved. Details of the above study is reported in [34]. 


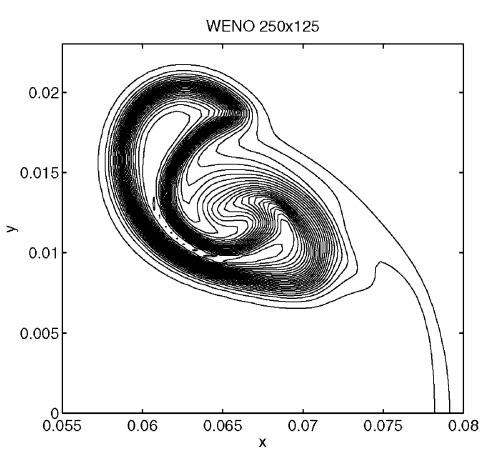

(a)

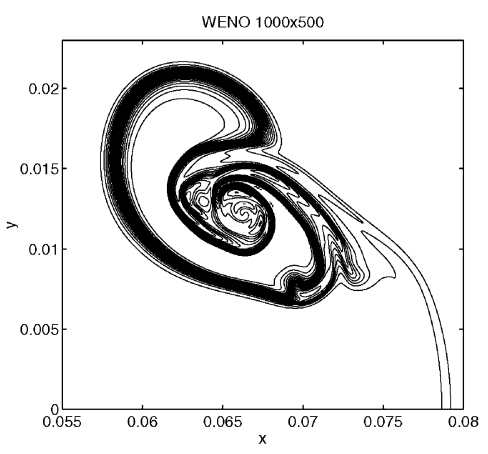

(c)

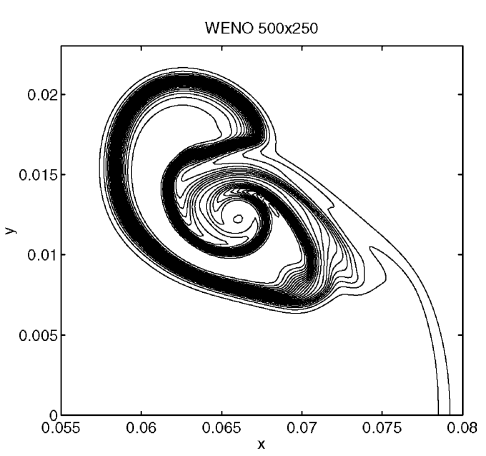

(b)

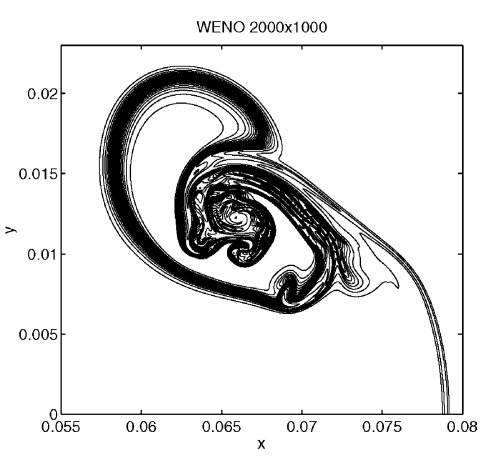

(d)

Fig. 14. Grid refinement of the WENO5-RK4 scheme. Hydrogen mass fraction contours at time $60 \mu$ s. (a) WENO5-RK4, $250 \times 125$ grid; (b) WENO5-RK4, $500 \times 250$ grid; (c) WENO5-RK4, $1000 \times 500$ grid; (d) WENO5-RK4, $2000 \times 1000$ grid.

\section{Computer implementation}

The computations presented here would not have been possible without use of high performance supercomputers. Most of the computations were done on a 512 processor SGI Origin2000 at NASA Ames Research Center. Some computations were done on a 300 processor IBM/SP2 at the Center for Parallel Computers (PDC) in Stockholm, Sweden. Our code is parallelized using the Message Passing Interface (MPI) library. The code automatically divides the computational domain into equal sized patches. Sufficient overlap of points between processors is allocated to allow the sometimes wide stencils which occur in high order difference methods. The computational domain is simple, and the numerical method is explicit, which means that it is easy to obtain good parallel performance. The few global operations, such as determination of the time step, are done through calls to reduction routines in MPI.

Computation times ranged from a few minutes on 10 processors for the smallest problems, to as much as $20 \mathrm{~h}$ of computing time on 256 processors for the largest computations. The different numerical methods were implemented with some care to have an efficient computation. For the WENO5 scheme, the number of operations in our implementation was approximately the same as the number given in [19]. The computational times were as follows. The ACM66-RK4 method had approximately the same CPU time as the second-order TVD scheme (MUSCL-RK2 and TVD-RK2). The wavelet version of the filter (WAV66RK4) required almost the same CPU time as the original ACM66-RK4 scheme. The WENO5-RK4 scheme consumed 2.5 times the CPU time of the MUSCL-RK2 and TVD-RK2. The measured times are for 

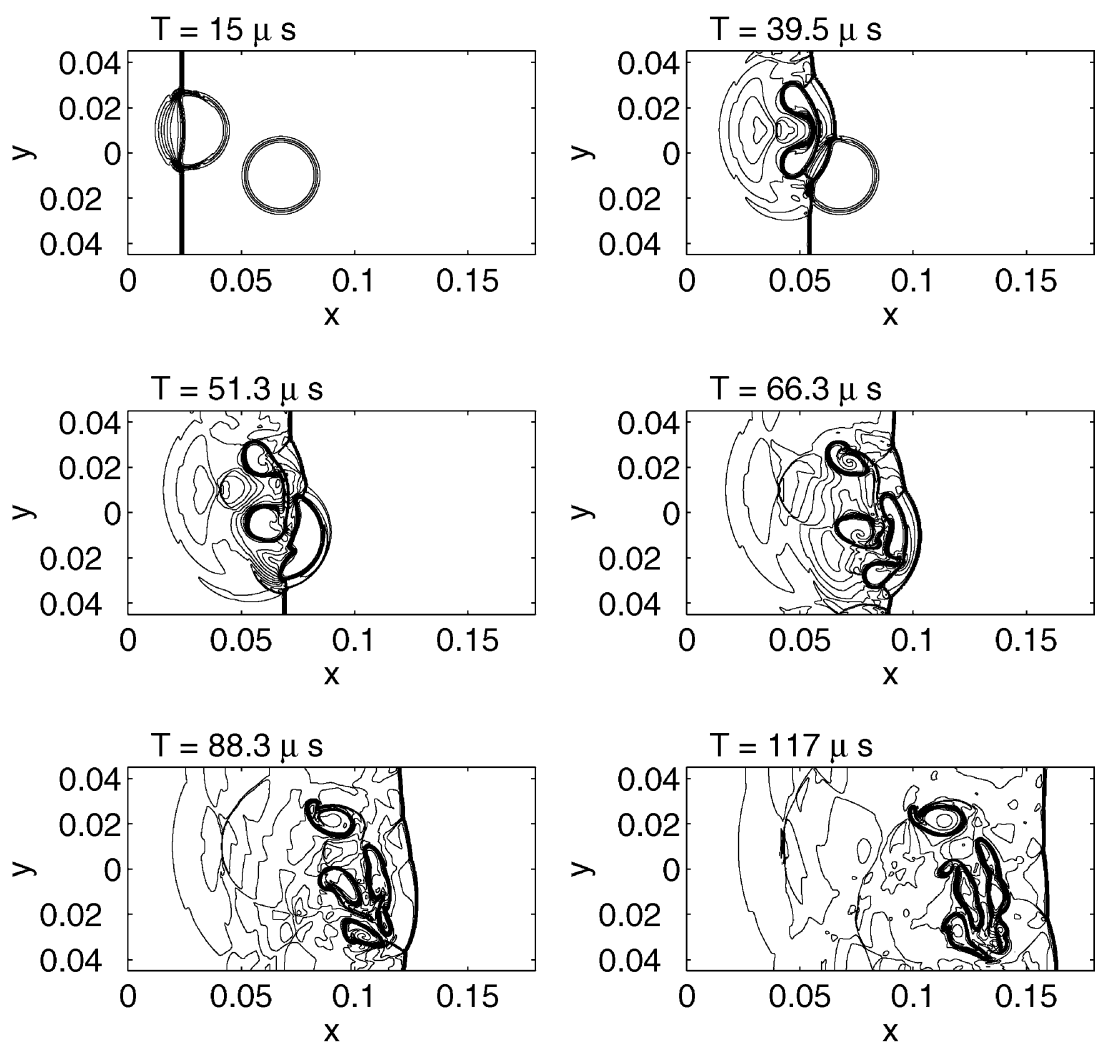

Fig. 15. Density contours by ACM66-RK4, using a $500 \times 250$ grid. Time evolution of a planar Mach 2 shock in air interacting with two initially non-aligned circular hydrogen bubbles.

computing one time step, and the total number of time steps required to reach to the same physical time unit by each methods has not been taken into consideration. The number of flux evaluations differ, since the WENO5-RK4, ACM66-RK4 and WAV66-RK4 schemes are integrated by a four stage fourth-order Runge-Kutta method (i.e., four viscous and inviscid flux evaluations), while for the MUSCL-RK2 and TVD-RK2 schemes, the fluxes are only evaluated twice by the second-order Runge-Kutta time integrator. The wavelet filter leads to a difference operator which has a stencil width which increases rapidly as the number of multiresolution levels of the wavelet increases. In the present implementation, the number of wavelet levels is small, and a sufficient interprocessor overlap is made. It is, however, possible to implement the wavelet algorithm more generally with a FFT-like butterfly communication pattern. This is a topic of current investigations.

The efficiency of WAV66-RK4 was improved by not computing the filter terms when the sensor was equal to zero. Due to the complexity in programming, this was not implemented, and could give some further CPU speedup for the ACM66-RK4 and WAV66-RK4 schemes.

We give in Tables 1-3, the CPU time required to take one complete time step for the viscous shock tube problem, with Reynolds number 1000, for different problem sizes. The program was run on an IBM/SP2 computer with Power 2 processors, having a clock frequency of $160 \mathrm{MHz}$, and $256 \mathrm{Mbytes}$ RAM memory. The processors communicate through a switch, which has a bandwidth of 110 Mbytes per second. The tables show the relative performance of the methods. However, many of the computations were run on an SGI Origin 2000 computer, using 128 processors. Conclusions about total computational times might not correspond to what was actually obtained. 
Table 1

CPU seconds per time step for TVD-RK at different grid sizes

\begin{tabular}{llll}
\hline Number of processors & $500 \times 250$ & $1000 \times 500$ & $2000 \times 1000$ \\
\hline 16 & 0.25 & 1.3 & - \\
32 & 0.15 & 0.70 & 2.9 \\
64 & 0.11 & 0.30 & 1.4 \\
\hline
\end{tabular}

Table 2

CPU seconds per time step for ACM66-RK4 at different grid sizes

\begin{tabular}{llll}
\hline Number of processors & $500 \times 250$ & $1000 \times 500$ & $2000 \times 1000$ \\
\hline 16 & 0.25 & 1.25 & - \\
32 & 0.15 & 0.51 & 1.9 \\
64 & 0.10 & 0.28 & 1.0 \\
\hline
\end{tabular}

Table 3

CPU seconds per time step for WAV66-RK4 at different grid sizes

\begin{tabular}{llll}
\hline Number of processors & $500 \times 250$ & $1000 \times 500$ & $2000 \times 1000$ \\
\hline 16 & 0.21 & 1.0 & - \\
32 & 0.12 & 0.41 & 1.5 \\
64 & 0.08 & 0.21 & 0.85 \\
\hline
\end{tabular}

\section{Conclusions}

We have shown that the recently proposed ACM and wavelet filter schemes of using sixth-order central spatial differencing as the base scheme (ACM66 and WAV66) are more efficient and more accurate than the fifth-order WENO scheme (WENO5) for the low Reynolds number flow. For high Reynolds number and/ or very strong inviscid shock computations, the filter method might be unstable with the use of compressive limiters. We are not certain if this is due to the scheme or due to the instability of the flow in the case of the high Reynolds problem. One remedy is to use a more diffusive limiter for the filter. Another remedy is to use the filter as the regular numerical dissipation for the central scheme. If one were to use the filter numerical fluxes as a better adaptive numerical dissipation control to be used with the central scheme for each stage of the Runge-Kutta method, the required CPU time would be comparable to that for the WENO5 scheme but a more accurate solution could be realized. We have performed grid convergence studies of flows with complex structures. It turned out that schemes of order 5 and 6 can capture the solution on a coarser grid than the one necessary with a standard second-order scheme. However, the improvement from using high order methods for rapidly developing complex stiff problems is not as dramatic as it is for simple test problems, long time wave propagations, or low Mach number compressible turbulence computations. With many levels of grid refinement, grid convergence was not reached by the majority of the flow configurations considered except for the shock/shear/boundary-layer interaction using $R e=200$. The present conclusion is based on the two test models, a comparison using a uniform Cartesian grid, and the convergence trends of the different methods. The uncertainty of the $R e=1000$ shock/shear/boundary-layer case makes it difficult to assess the accuracy and robustness of these schemes. For the combustion model it is even more difficult to judge their performance. To reduce the grid size while still obtaining well-resolved simulations, a more relevant comparison for the application of practical computations is to incorporate the dual purpose adaptive property of the wavelet sensor, namely, grid adaptation and dynamic numerical dissipation control indicator. This is a topic of current investigation. 
The results shown here were all computed on a uniform grid. When using a high order method on a curvilinear grid, smoothness requirements for the grid are severe. In practise only domains where analytical expressions for the boundaries are known can be used. Whether high order schemes are advantageous on curvilinear grids can not be deduced from this work. It is a topic for future investigations.

\section{Acknowledgements}

The authors would like to thank Wai Sun Don for providing his spectral chemical reaction code as a guide to build our research code for the supersonic reactive flow study. Thanks also to Jahed Djomehri and Neil Sandham for the initial implementation of the shock/shear/boundary-layer problem on the NASA Ames CRAY C90 machine. The generous help from Jahed Djomehri on debugging of the parallel codes, the computer resource support from COSMO on the NASA Ames 512 processors Origin 2000 machine, and the permission from Chuck Niggley on the special queue privilege to speed up the turnaround time are gratefully acknowledged. Without the special queue privilege, it would not have been possible to complete the present study in a reasonable amount of time. Special thanks to Virginie Daru for providing a copy of her shock/shear/boundary-layer code as a guide for the physical problem implementation.

\section{References}

[1] P. Alpert, Implicit filtering in conjunction with explicit filtering, J. Comput. Phys. 44 (1981) 212-219.

[2] D.L. Brown, M.L. Minion, Performance of under-resolved two-dimensional incompressible flow simulations, J. Comput. Phys. 122 (1995) 165-183.

[3] D.L. Brown, L. Margolin, D. Sharp, A.A. White, Predictability of Complex Phenomena, A White Paper, Los Alamos National Laboratory reprint, 1997.

[4] J. Nordström, M.H. Carpenter, Boundary and interface conditions for high-order finite-difference methods applied to the Euler and Navier-Stokes equations, J. Comput. Phys. 148 (1999) 621-645.

[5] M.H. Carpenter, D. Gottlieb, S. Abarbanel, Time-stable boundary conditions for finite-difference schemes solving hyperbolic systems: methodology and application to high-order compact schemes, J. Comput. Phys. 111 (1994) $220-236$.

[6] M.H. Carpenter, D. Gottlieb, S. Abarbanel, W.-S. Don, The theoretical accuracy of Runge-Kutta time discretizations for initial value problem: a study of the boundary error, SIAM J. Sci. Comput. 16 (1995) 1241-1252.

[7] V. Daru, C. Tenaud, Evaluation of TVD high resolution schemes for unsteady viscous shocked flows, Comput. Fluids 30 (2000) 89-113.

[8] W.S. Don, C.B. Quillen, Numerical simulation of shock-cylinder interactions, J. Comput. Phys. 122 (1995) $244-265$.

[9] W.S. Don, D. Gottlieb, Spectral simulation of supersonic reactive flows, SIAM J. Numer. Anal. 35 (1998) $2370-2384$.

[10] D.V. Gaitone, M.R. Visbal, Further Development of a Navier-Stokes Solution Procedure based on Higher-order Formulas, AIAA Paper 99-0557, Reno, NV, 1999.

[11] M. Gerritsen, P. Olsson, Designing an efficient solution strategy for fluid flows, J. Comput. Phys. 129 (1996) $245-262$.

[12] D.F. Griffiths, P.K. Sweby, H.C. Yee, On spurious asymptotic numerical solutions of explicit Runge-Kutta schemes, IMA J. Numer. Anal. 12 (1992) 319-338.

[13] J. Glimm, D. Sharp, Prediction and the Quantification of Uncertainty, Los Alamos National Laboratory LAUR-98-3391, 1998.

[14] P.M. Gresho, D.K. Gartling, J.R. Torczynski, K.A. Cliffe, K.H. Winters, T.J. Garrett, A. Spence, J.W. Goodrich, Is the steady viscous incompressible two-dimensional flow over a backward-facing step at $R e=800$ stable? Int. J. Numer. Meth. Fluids 17 (1993) 501-541.

[15] B. Gustafsson, H.-O. Kreiss, J. Oliger, Time Dependent Problems and Difference Methods, Wiley, New York, 1995.

[16] A. Harten, The artificial compression method for computation of shocks and contact discontinuities: III. Self-adjusting hybrid schemes, Math. Comp. 32 (1978) 363-389.

[17] A. Harten, On the symmetric form of systems for conservation laws with entropy, J. Comput. Phys. 49 (1983) $151-164$.

[18] M. Johansson, Loss of High Order Spatial Accuracy due to Boundary Error Caused by Runge-Kutta Time Integration, Technical Report 2000-013, Department of Information Technology, Uppsala University, May 2000.

[19] G.-S. Jiang, C.-W. Shu, Efficient Implementation of Weighted ENO Schemes, ICASE Report No. 95-73, 1995. 
[20] A. Lafon, H.C. Yee, Dynamical approach study of spurious steady-state numerical solutions for nonlinear differential equations, part III: the effects of nonlinear source terms in reaction-convection equations, Comp. Fluid Dyn. 6 (1996) 1-36.

[21] A. Lafon, H.C. Yee, Dynamical approach study of spurious steady-state numerical solutions of nonlinear differential equations, part IV: stability vs. numerical treatment of nonlinear source terms, Comput. Fluid Dyn. 6 (1996) 89-123.

[22] S.A. Lele, Compact finite difference schemes with spectral-like resolution, J. Comput. Phys. 103 (1992) 16-42.

[23] R.J. LeVeque, H.C. Yee, A study of numerical methods for hyperbolic conservation laws with stiff source terms, J. Comput. Phys. 86 (1990) 187-210.

[24] K. Mattsson, Imposing Boundary Conditions with the Injection, the Projection and the Simultaneous Approximation Term Methods, Proceedings of the First International Conference on CFD, July 10-14, Kyoto, Japan, 2000.

[25] B.J. McBride, S. Heimel, J.G. Ehlers, S. Gorden, Thermodynamics Properties to $6000 \mathrm{~K}$ for 210 Substances Involving the first 18 elements, NASA SP-3002, 1963.

[26] M.L. Minion, D.L. Brown, Performance of under-resolved two-dimensional incompressible flow simulations II, J. Comput. Phys. 138 (1997) 734-765.

[27] W.L. Oberkampf, K.V. Diegert, K.F. Alvin, B.M. Rutherford, Variability, Uncertainty and Error in Computational Simulation, ASME Proceedings of the 7th AIAA/ASME Joint Thermophysics and Heat Transfer Conference, HTD-vol. 3572, 1998.

[28] P. Olsson, J. Oliger, Energy and Maximum Norm Estimates for Nonlinear Conservation Laws, RIACS Technical Report 94.01, 1994.

[29] P. Olsson, Summation by parts, projections, and stability. I, Math. Comp. 64 (1995) 1035-1065.

[30] P. Olsson, Summation by parts, projections, and stability. II, Math. Comp. 64 (1995) 1473-1493.

[31] P. Olsson, Summation by Parts, Projections, and Stability. III, RIACS Technical Report 95.06, 1995.

[32] N.D. Sandham, H.C. Yee, Entropy Splitting for High Order Numerical Simulation of Compressible Turbulence, RIACS Technical Report 00.10, June, 2000, Proceedings of the 1st International Conference on CFD, July 10-14, Kyoto, Japan, 2000.

[33] B. Sjögreen, H.C. Yee, Multiresolution Wavelet based Adaptive Numerical Dissipation Control for Shock-Turbulence Computation, RIACS Report 01.01, NASA Ames Research Center, October 2000.

[34] B. Sjögreen, H.C. Yee, Low Dissipative High Order Numerical Simulations of Supersonic Reactive Flows, RIACS Report 01-017, NASA Ames research center, May 2001, Proceedings of the ECCOMAS Computational Fluid Dynamics Conference 2001, Swansea, Wales, UK, September 4-7, 2001.

[35] Special Section of the AIAA Journal, Credible computational fluid dynamics simulation, AIAA J. 36 (1998) 665-759.

[36] B. Strand, Summation by parts for finite difference approximations for d/dx, J. Comput. Phys. 110 (1994) $47-67$.

[37] B. Strand, High-Order Difference Approximations for Hyperbolic Initial Boundary Value Problems, PhD thesis, Uppsala University, Department of Scientific Computing, 1996.

[38] R.A. Svehla, Estimated Viscosities and Thermal Conductivities of Gases at High Temperatures, NASA Report TR R-132, 1962.

[39] J.R. Torczynski, A grid refinement study of two-dimensional transient flow over a backward-facing step using a spectral-element method, in: J.C. Dutton, L.P. Purtell (Eds.), Separated Flows, FED-vol. 149, ASME, New York, 1993.

[40] T.G. Trucano, Prediction and Uncertainty in Computational Modeling of Complex Phenomena: A Whitepaper, Sandia Report, SAND98-2776, 1998.

[41] M. Vinokur, H.C. Yee, Extension of Efficient Low Dissipative High Order Schemes for 3-D Curvilinear Moving Grids, NASA TM 209598, June 2000.

[42] R. Vichnevetsky, Numerical Filtering for Partial Differencing Equations, Numerical Applications Memorandum, Rutgers University, NAM 156, 1974.

[43] C.R. Wilke, A viscosity equation for gas mixtures, Chem. Phys. 18 (1950) 517-519.

[44] H.C. Yee, P.K. Sweby, D.F. Griffiths, Dynamical approach study of spurious steady-state numerical solutions for nonlinear differential equations, part I: the dynamics of time discretizations and its implications for algorithm development in computational fluid dynamics, NASA TM-102820, April 1990, J. Comput. Phys. 97 (1991) 249-310.

[45] H.C. Yee, P.K. Sweby, Dynamics of Numerics and Spurious Behaviors in CFD Computations, Keynote paper, 7th ISCFD Conference, September 15-19, 1997, Beijing, China, RIACS Technical Report 97.06, June 1997.

[46] H.C. Yee, P.K. Sweby, Some Aspects of Numerical Uncertainties in Time Marching to Steady-State Computations, AIAA-962052, 27th AIAA Fluid Dynamics Conference, June 18-20, 1996, New Orleans, LA, AIAA J., 36 (1998) $712-724$.

[47] H.C. Yee, J.R. Torczynski, S.A. Morton, M.R. Visbal, P.K. Sweby, On Spurious Behavior of CFD Simulations, AIAA 97-1869, Proceedings of the 13th AIAA Computational Fluid Dynamics Conference, June 29-July 2, 1997, Snowmass, CO., also International J. Numer. Meth. Fluids 30 (1999) 675-711.

[48] H.C. Yee, N.D. Sandham, M.J. Djomehri, Low Dissipative high order shock-capturing methods using characteristic-based filters, J. Comput. Phys. 150 (1999) 199-238.

[49] H.C. Yee, M. Vinokur, M.J. Djomehri, Entropy splitting and numerical dissipation, J. Comput. Phys. 162 (2000) $33-81$. 